\title{
Prethermal Phases of Matter Protected by Time-Translation Symmetry
}

\author{
Dominic V. Else, ${ }^{1}$ Bela Bauer, ${ }^{2}$ and Chetan Nayak ${ }^{2,1}$ \\ ${ }^{1}$ Physics Department, University of California, Santa Barbara, California 93106, USA \\ ${ }^{2}$ Station Q, Microsoft Research, Santa Barbara, California 93106-6105, USA \\ (Received 27 September 2016; revised manuscript received 24 January 2017; published 7 March 2017) \\ In a periodically driven (Floquet) system, there is the possibility for new phases of matter, not present in \\ stationary systems, protected by discrete time-translation symmetry. This includes topological phases \\ protected in part by time-translation symmetry, as well as phases distinguished by the spontaneous breaking \\ of this symmetry, dubbed "Floquet time crystals." We show that such phases of matter can exist in the \\ prethermal regime of periodically driven systems, which exists generically for sufficiently large drive \\ frequency, thereby eliminating the need for integrability or strong quenched disorder, which limited \\ previous constructions. We prove a theorem that states that such a prethermal regime persists until times \\ that are nearly exponentially long in the ratio of certain couplings to the drive frequency. By similar \\ techniques, we can also construct stationary systems that spontaneously break continuous time-translation \\ symmetry. Furthermore, we argue that for driven systems coupled to a cold bath, the prethermal regime \\ could potentially persist to infinite time.
}

DOI: 10.1103/PhysRevX.7.011026

Subject Areas: Condensed Matter Physics

\section{INTRODUCTION}

Much of condensed matter physics revolves around determining which distinct phases of matter can exist as equilibrium states of physical systems. Within a phase, the properties of the system vary continuously as external parameters are varied, while different phases are separated by phase transitions, at which the properties change abruptly. An extremely rich set of observed phases can be characterized by symmetry. The best-known example is spontaneous symmetry breaking, which results in the equilibrium state of the system being less symmetrical than the Hamiltonian. More recently, a set of uniquely quantum phases-symmetry-protected topological (SPT) phases [1-19], including topological insulators [20,21], and symmetry-enriched topological (SET) phases [22-28] has been discovered. These phases, while symmetric, manifest the symmetry in subtly anomalous ways and are distinct only as long as the symmetry is preserved. We can collectively refer to these three classes of phases as symmetry-protected phases of matter.

Thus far, the concept of symmetry-protected phases of matter has not been as successful in describing systems away from equilibrium. Recently, however, it was realized that certain periodically driven "Floquet" systems can exhibit distinct phases, akin to those of equilibrium systems [29]. In this paper, we show that there is, in fact, a very

Published by the American Physical Society under the terms of the Creative Commons Attribution 4.0 International license. Further distribution of this work must maintain attribution to the author(s) and the published article's title, journal citation, and DOI. general set of nonequilibrium conditions under which such phases can arise, due to a remarkable phenomenon called "prethermalization." In Floquet systems, prethermalization occurs when a time-dependent change of basis removes all but a small residual time dependence from the Hamiltonian and thus allows the properties of the system to be mapped approximately onto those of a system in thermal equilibrium. The residual time dependence is nearly exponentially small in a large parameter $\alpha$ of the original Hamiltonian of the system. One can then talk about a "prethermal regime" in which the system reaches a thermal equilibrium state with respect to the approximate effective time-independent Hamiltonian that results from neglecting the small residual time dependence. In this regime, the system can exhibit phases and phase transitions analogous to those seen in thermal equilibrium, such as symmetry-protected phases. Nevertheless, in the original nonrotating frame, the system remains very far from thermal equilibrium with respect to the instantaneous Hamiltonian at any given time. After the characteristic time $t_{*}$, which is nearly exponentially long in the large parameter $\alpha$, other physics (related to the residual time dependence) takes over.

In this paper, we show that prethermal systems can also exhibit phases of matter that cannot exist in thermal equilibrium. These novel phases can also be understood as symmetry-protected phases but of a variety that cannot occur in thermal equilibrium: These phases are protected by discrete time-translation symmetry. While these phases include topological phases protected by time-translation symmetry [30-33], perhaps the most dramatic are "time crystals" that spontaneously break time-translation symmetry. The idea of time crystals that spontaneously break continuous time-translation symmetry was first proposed 
by Wilczek and Shapere [34,35]; however, finding a satisfactory equilibrium model has proven difficult, and some no-go theorems exist [36-42]. In this paper, we construct prethermal "Floquet time crystals," which spontaneously break the discrete time-translation symmetry of periodically driven systems [43]. (For an alternative view of such systems that focuses on other symmetries of the discrete time-translation operator, see Refs. [29,44,45].) Floquet time crystals are the focus of this paper, but as a by-product of our analysis, we also find prethermal-i.e., nonequilibrium-time crystals that spontaneously break continuous time-translation symmetry. We also construct SPT and SET phases protected by discrete time-translation symmetry.

Periodically driven systems have long been considered an unlikely place to find interesting phases of matter and phase transitions since generic driven closed systems will heat up to infinite temperature [46-48]. It has been known that the heating problem can be avoided [49-53] if the system is integrable or if the system has sufficiently strong quenched disorder that it undergoes many-body localization (MBL) [54-63]. However, integrability relies on finetuning, and MBL requires the system to be completely decoupled from the environment [64-72]. Furthermore, the disorder must be sufficiently strong, which may be difficult to realize in an experiment but does not constitute fine-tuning.

The central result of this paper is therefore to show that prethermalization makes it possible for nonequilibrium phases protected by time-translation symmetry to occur in more generic nonequilibrium systems without the need for fine-tuning, strong disorder, or complete decoupling from the environment. Remarkably, these nonequilibrium phases and phase transitions, which have no direct analogues in thermal equilibrium, have a mathematical formulation that is identical to that of equilibrium phases, though with a different physical interpretation. Since MBL is not a requirement, it is conceivable that prethermal timetranslation protected phases could survive the presence of coupling to an environment. In fact, we will discuss a plausible scenario by which these phases can actually be stabilized by coupling to a sufficiently cold thermal bath, such that the system remains in the prethermal regime even at infinite time.

The structure of the paper is as follows. In Sec. II, we state our main technical result. In Sec. III, we apply this to construct prethermal Floquet time crystals, which spontaneously break discrete time-translation symmetry. In Sec. IV, we show that a continuous time-translation symmetry can also be spontaneously broken in the prethermal regime for a system with a time-independent Hamiltonian. In Sec. V, we outline how our methods can also be applied to construct SPT and SET phases protected by time-translation symmetry. In Sec. VI, we discuss what we expect to happen for nonisolated systems coupled to a cold thermal bath. Finally, we discuss implications and interpretations in Sec. VII.

\section{PRETHERMALIZATION RESULTS}

The simplest incarnation of prethermalization occurs in periodically driven systems when the driving frequency $\nu$ is much larger than all of the local energy scales of the instantaneous Hamiltonian [73-77] (see also Refs. [78-80] for numerical results). The key technical result of our paper will be a theorem generalizing these results to other regimes in which the driving frequency is not greater than all the local scales of the Hamiltonian, but there is nevertheless some separation of energy scales. This will allow us to show that time-translation protected phases can exist in the prethermal regime. More precisely, in the models that we construct, one local coupling strength is large and the others are small; the drive frequency is large compared to the small couplings, and the parameter $\alpha$ is the ratio of the drive frequency to the largest of the small local couplings. The term in the Hamiltonian with large coupling must take a special form, essentially that of a symmetry generator, which allows it to avoid heating the system.

Accordingly, we consider a time-dependent Hamiltonian of the form $H(t)=H_{0}(t)+V(t)$, where $H_{0}(t)$ and $V(t)$ are periodic with period $T$. We assume that $\lambda T \ll 1$, where $\lambda$ is the local energy scale of $V$. We further assume that $H_{0}(t)$ has the property that it generates a trivial time evolution over $N$ time cycles: $U_{0}(N T, 0)=$ $U_{0}(T, 0)^{N}=1$, where

$$
\begin{aligned}
U_{0}\left(t_{2}, t_{1}\right) & =\mathcal{T} \exp \left(-i \int_{t_{1}}^{t_{2}} H_{0}(t)\right) d t, \\
\mathcal{T} & =\text { time ordering. }
\end{aligned}
$$

We claim that such a time evolution will exhibit prethermalizing behavior for $\lambda T \ll 1 / N$ even if the local energy scale of $H_{0}(t)$ is comparable to $1 / T$. In other words, such a system exhibits prethermalizing behavior when the frequency is large compared to some of the couplings [those in $V(t)]$ but not others [those in $H_{0}(t)$ ], as promised in the Introduction.

An easy way to see that this claim is true is to work in the interaction picture (treating $V$ as the "interaction"). Then, we see that the time evolution of the total Hamiltonian $H(t)$ over $N$ time cycles is given by

$$
U(N T, 0)=\mathcal{T} \exp \left(-i \int_{0}^{N T} V^{\mathrm{int}}(t) d t\right)
$$

where $V^{\text {int }}(t)=U_{0}(0, t)^{\dagger} V(t) U_{0}(0, t)$ is the representation of $V(t)$ in the interaction picture, and $U_{0}(0, N T)=1$ ensures that the time evolution operator Eq. (2) is the same in the interaction and Schrödinger pictures. If we rescale time as $t \rightarrow t / \lambda$, then Eq. (2) describes a system 
being driven at the large frequency $\nu=1 /(\lambda N T)$ by a drive of local strength 1, which by the results of Refs. [73-77], will exhibit prethermalizing behavior for $\nu \gg 1$.

On the other hand, since the above argument for prethermalization required coarse-graining the time period from $T$ to $N T$, it prevents us from identifying phases of matter, such as time crystals or Floquet SPT phases, that are protected by time-translation symmetry. The problem is that the time-translation symmetry by $T$ is what allows different phases of matter to be sharply distinguished. This symmetry is still present, of course (because the coarsegraining is a feature of our description of the system, not the system itself), but it is no longer manifest. Therefore, it is not at all transparent how to understand the different phases of matter in this picture.

In order to proceed further, we need a new approach. In this paper, we develop a new formalism that analyzes $U(T, 0)$ itself rather than $U(N T, 0)$, allowing the effects of time-translation symmetry to be seen in a transparent way. Our central tool is a theorem that we will prove, substantially generalizing those of Abanin et al. [74]. A more precise version of our theorem will be given momentarily, and the proof will be given in Appendix A; the theorem essentially states that there exists a time-independent local unitary rotation $\mathcal{U}$ such that $U_{\mathrm{f}} \approx \tilde{U}_{\mathrm{f}}=\mathcal{U}^{\dagger}\left(X e^{-i D T}\right) \mathcal{U}$, where $X=U_{0}(T, 0)$ is the time evolution of $H_{0}$ over one time cycle, and $D$ is a quasilocal Hamiltonian that commutes with $X$. The dynamics at stroboscopic times are well approximated by $\tilde{U}_{\mathrm{f}}$ for times $t \ll t_{*}$, where $t_{*}=e^{O\left(1 /\left(\lambda T[\log (1 / \lambda T)]^{3}\right)\right)}$. This result combines ideas in Ref. [74] about (1) the high-frequency limit of driven systems and (2) approximate symmetries in systems with a large separation of scales. Recall that, in the high-frequency limit of a driven system, the Floquet operator can be approximated by the evolution (at stroboscopic times) due to a time-independent Hamiltonian, $U_{\mathrm{f}} \approx \exp \left(-i T H_{\text {eff }}\right)$. Meanwhile, in a static system with a large separation of scales, $H=-u L+D_{0}$, where $u$ is much larger than the couplings in $D_{0}$ but $\left[L, D_{0}\right] \neq 0$, Ref. [74] shows that there is a unitary transformation $\mathcal{U}$ such that $\mathcal{U} H \mathcal{U}^{\dagger} \approx-u L+D$, where $[L, D]=0$; i.e., the system has an approximate symmetry generated by $\mathcal{U}^{\dagger} L \mathcal{U}$. Our theorem states that, after a time-independent local unitary change of basis, a periodic Hamiltonian $H(t)=H_{0}(t)+V(t)$, with $H_{0}(t)$ satisfying the condition given above, can be approximated, as far as the evolution at stroboscopic times is concerned, by a binary drive that is composed of two components: (1) the action of $H_{0}(t)$ over one cycle, namely, $U_{0}(T, 0)$, and (2) a static Hamiltonian that is invariant under the symmetry generated by $U_{0}(T, 0)$.

These results might seem surprising because they imply that the evolution over one time period commutes with a symmetry $X=U_{0}(T, 0)$ (or $\mathcal{U} X \mathcal{U}^{\dagger}$ in the original basis), despite the fact that the microscopic time-dependent Hamiltonian $H(t)$ had no such symmetry. We interpret this "hidden" symmetry as a shadow of the discrete timetranslation symmetry. (For example, the evolution over $N$ time periods also commutes with $\mathcal{U} X \mathcal{U}^{\dagger}$, but if we add weak $N T$-periodic perturbations to break the discrete timetranslation symmetry, then this is no longer the case.) Thus, our theorem precisely allows us to get a handle on the implications of discrete time-translation symmetry. Compare Ref. [45], where a similar "hidden" symmetry was constructed for many-body-localized Floquet time crystals.

The preceding paragraphs summarize the physical meaning of our theorem. A more precise statement of the theorem, although it is a bit more opaque physically, is useful because it makes the underlying assumptions manifest. The statement of the theorem makes use of an operator norm $\|O\|_{n}$ that measures the average over one Floquet cycle of the size of the local terms whose sum makes up a Hamiltonian; the subscript $n$ parametrizes the extent to which the norm suppresses the weight of operators with larger spatial support. An explicit definition of the norm is given in Appendix A. The theorem states the following.

Theorem 1. Consider a periodically driven system with a Floquet operator:

$$
U_{f}=\mathcal{T} \exp \left(-i \int_{0}^{T} H(t) d t\right)
$$

where $H(t)=H_{0}(t)+V(t)$, and $X \equiv U_{0}(0, T)$ satisfies $X^{N}=1$ for some integer $N$. We assume that $H_{0}(t)$ can be written as a sum $H_{0}(t)=\sum_{i} h_{i}(t)$ of terms acting only on single sites $i$. Define $\lambda \equiv\|V\|_{1}$. Assume that

$$
\lambda T \leq \frac{\gamma \kappa_{1}^{2}}{N+3}, \quad \gamma \approx 0.14
$$

Then, there exists a (time-independent) unitary $\mathcal{U}$ such that

$$
\mathcal{U} U_{f} \mathcal{U}^{\dagger}=X \mathcal{T} \exp \left(-i \int_{0}^{T}[D+E+V(t)] d t\right)
$$

where $D$ is local and $[D, X]=0 ; D, E$ are independent of time; and

$$
\begin{aligned}
\|V\|_{n_{*}} & \leq \lambda\left(\frac{1}{2}\right)^{n_{*}}, \\
\|E\|_{n_{*}} & \leq \lambda\left(\frac{1}{2}\right)^{n_{*}} .
\end{aligned}
$$

The exponent $n_{*}$ is given by 


$$
\begin{aligned}
& n_{*}=\frac{\lambda_{0} / \lambda}{\left[1+\log \left(\lambda_{0} / \lambda\right)\right]^{3}}, \\
& \lambda_{0}=\frac{\left(\kappa_{1}\right)^{2}}{72(N+3)(N+4) T} .
\end{aligned}
$$

Furthermore,

$$
\|D-\bar{V}\|_{n_{*}} \leq \mu\left(\lambda^{2} / \lambda_{0}\right), \quad \mu \approx 2.9,
$$

where

$$
\begin{aligned}
\bar{V} & =\frac{1}{N T} \int_{0}^{N T} V^{\mathrm{int}}(t) d t \\
& =\frac{1}{N} \sum_{k=0}^{N-1} X^{-k}\left(\frac{1}{T} \int_{0}^{T} V^{\mathrm{int}}(t) d t\right) X^{k} .
\end{aligned}
$$

The proof is given in Appendix A. The statement of the theorem makes use of a number $\kappa_{1}$. It is chosen so that $\|H\|_{1}$ is finite; the details can be found in Appendix A, where the norm is given.

Unpacking the theorem a bit in order to make contact with the discussion above, we see that it states that there is a time-independent unitary operator $\mathcal{U}$ that transforms the Floquet operator into the form $X e^{-i D T}$ with $[D, X]=0$ and local $D$, up to corrections that are exponentially small in $n_{*} \sim 1 /\left(\lambda T[\ln (1 / \lambda T)]^{3}\right)$. These "error terms" fall into two categories: time-independent terms that do not commute with $X$, which are grouped into $E$; and time-dependent terms, which are grouped into $V(t)$. Both types of corrections are exponentially small in $n_{*}$. Since they are exponentially small, $\|E\|_{n_{*}},\|V\|_{n_{*}} \sim(1 / 2)^{n_{*}}$, these terms do not affect the evolution of the system until exponentially long times, $t_{*} \sim e^{C n_{*}}$ (for some constant $C$ ). It is not possible to find a time-independent unitary transformation that exactly transforms the Floquet operator into the form $X e^{-i D T}$ because the system must, eventually, heat up to infinite temperature and the true Floquet eigenstates are infinitetemperature states, not the eigenstates of an operator of the form $X e^{-i D T}$ with local $D$. In the interim, however, the approximate Floquet operator $X e^{-i D T}$ leads to Floquet time-crystal behavior, as we discuss in the next section.

The proof of Theorem 1 constructs $\mathcal{U}$ and $D$ through a recursive procedure, which combines elements of the proofs of prethermalization in driven and undriven systems given by Abanin et al. [74].

In the case of prethermal undriven systems, the theorem we need has essentially already been given in Ref. [74], but we will restate the result in a form analogous with Theorem 1, which entails some slightly different bounds (however, they are easily derivable using the techniques of Ref. [74]).
Theorem 2. Consider a time-independent Hamiltonian $H$ of the form

$$
H=-u L+V,
$$

where $e^{2 \pi i L}=1$. We assume that $L$ can be written as a sum $L=\sum_{i} L_{i}$ of terms acting only on single sites $i$. Define $\lambda \equiv\|V\|_{1}$, and assume that

$$
\lambda / u \leq \gamma \kappa_{1}^{2}, \quad \gamma \approx 0.14
$$

Then, there exists a local unitary transformation $\mathcal{U}$ such that

$$
\mathcal{U} H \mathcal{U}^{\dagger}=-u L+D+\hat{V},
$$

where $[L, D]=0$ and $\hat{V}$ satisfies

$$
\|\hat{V}\|_{n_{*}} \leq \lambda\left(\frac{1}{2}\right)^{n_{*}}
$$

where

$$
n_{*}=\frac{\lambda_{0} / \lambda}{\left[1+\log \left(\lambda_{0} / \lambda\right)\right]^{3}}, \quad \lambda_{0}=\frac{u \kappa_{1}^{2}}{144} .
$$

Furthermore,

$$
\|D-\langle V\rangle\|_{n_{*}} \leq \mu\left(\lambda^{2} / \lambda_{0}\right), \quad \mu \approx 2.9 .
$$

Here, we have defined, following Ref. [74], the symmetrized operator $\langle V\rangle$ according to

$$
\langle V\rangle \equiv \int_{0}^{2 \pi} \frac{d \theta}{2 \pi} e^{i L \theta} V e^{-i L \theta},
$$

which, by construction, satisfies $[L,\langle V\rangle]=0$.

\section{PRETHERMALIZED FLOQUET TIME CRYSTALS}

\section{A. Basic picture}

The results of the previous section give us the tools we need to construct a model that is a Floquet time crystal in the prethermalized regime. Our approach is reminiscent of Ref. [45], where the Floquet-MBL time crystals of Ref. [43] were reinterpreted in terms of a spontaneously broken "emergent" $\mathbb{Z}_{2}$ symmetry. Here, emergent refers to the fact that the symmetry is, in some sense, hidden-its form depends on the parameters of the Hamiltonian in a manner that is not a priori known. Furthermore, it is not a symmetry of the Hamiltonian but rather a symmetry of the Floquet operator.

In particular, suppose that we have a model where we can set $X=\prod_{i} \sigma_{i}^{x}$. (Thus, $N=2$.) We then have $U_{\mathrm{f}} \approx \tilde{U}_{\mathrm{f}}=\mathcal{U}^{\dagger}\left(X e^{-i D T}\right) \mathcal{U}$, where the quasilocal Hamiltonian $D$, by construction, respects the Ising 
symmetry generated by $X$. This Ising symmetry corresponds to an approximate emergent symmetry $\mathcal{U} X \mathcal{U}^{\dagger}$ of $U_{\mathrm{f}}$ (the term emergent is used for the reason stated above, and approximate because it is an exact symmetry of $\tilde{U}_{\mathrm{f}}$, not $U_{\mathrm{f}}$, and therefore is approximately conserved for times $t \ll t_{*}$ ). Suppose that $D$ spontaneously breaks the symmetry $X$ below some finite critical temperature $\tau_{c}$. For example, working in two dimensions or higher, we could have $D=-J \sum_{\langle i, j\rangle} \sigma_{i}^{z} \sigma_{j}^{z}$ plus additional smaller terms of strength which break integrability. We are interested in the regime where the heating time $t_{*} \gg t_{\text {pre-thermal }}$, where $t_{\text {pre-thermal }}$ is the thermalization time of $D$.

Now, consider the time evolution $|\psi(t)\rangle$, starting from a given short-range correlated state $|\psi(0)\rangle$. We also define the rotated states $|\tilde{\psi}(t)\rangle=\mathcal{U}|\psi(t)\rangle$. At stroboscopic times $t=n T$, we find that $|\tilde{\psi}(n T)\rangle=\left(X e^{-i D T}\right)^{n}|\tilde{\psi}(0)\rangle$. Since $\left(X e^{-i D T}\right)^{2}=e^{-2 i D T}$, we see that at even multiples of the period, $t=2 n T$, the time evolution of $|\tilde{\psi}(t)\rangle$ is described by the time-independent Hamiltonian $D$. Thus, we expect that, after the time $t_{\text {pre-thermal }}$, the system appears to be in a thermal state of $D$ at temperature $\tau$. Thus, $|\tilde{\psi}(2 n T)\rangle\langle\tilde{\psi}(2 n T)| \approx \tilde{\rho}$, where $\tilde{\rho}$ is a thermal density matrix for $D$ at some temperature $\tau$, and the approximate equality means that the expectation values of local observables are approximately the same. Note that for $\tau<\tau_{c}$, the Ising symmetry of $D$ is spontaneously broken and $\tilde{\rho}$ must either select a nonzero value for the order parameter $M_{2 n}=\left\langle\sigma_{i}^{z}\right\rangle_{\tilde{\rho}}$ or have long-range correlations. The latter case is impossible given our initial state, as long-range correlations cannot be generated in finite time. Then, at odd times $t=(2 n+1) T$, we have

$$
\begin{aligned}
|\tilde{\psi}((2 n+1) T)\rangle\langle\tilde{\psi}((2 n+1) T)| & \approx\left(X e^{-i D T}\right) \tilde{\rho}\left(e^{i D T} X\right) \\
& =X \tilde{\rho} X
\end{aligned}
$$

(a) Time crystal

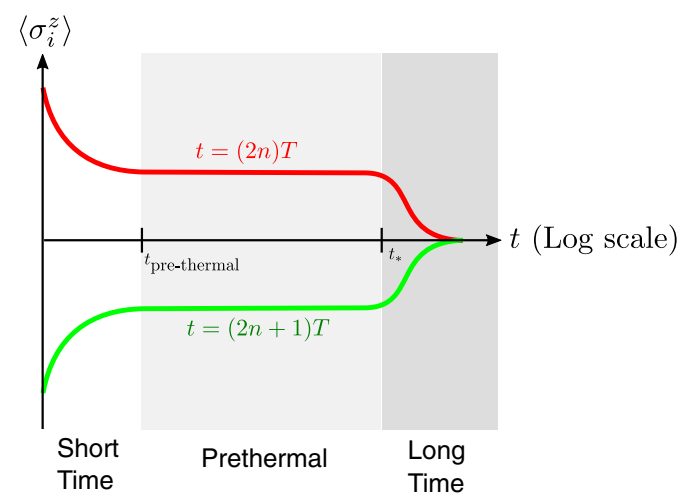

(since $\tilde{\rho}$ commutes with $D$ ). Therefore, at odd times, the order parameter

$$
M_{2 n+1}=\left\langle\sigma_{i}^{z}\right\rangle_{X \tilde{\rho} X}=-M_{2 n}
$$

Thus, the state of the system at odd times is different from the state at even times, and time translation by $T$ is spontaneously broken to time translation by $2 T$.

The above analysis took place in the frame rotated by $\mathcal{U}$. However, we can also consider the expectation values of operators in the original frame, for example, $\left\langle\psi(t)\left|\sigma_{i}^{z}\right| \psi(t)\right\rangle=\left\langle\tilde{\psi}(t)\left|\mathcal{U}^{\dagger} \sigma_{i}^{z} \mathcal{U}\right| \tilde{\psi}(t)\right\rangle$. The rotation $\mathcal{U}$ is close to the identity in the regime where the heating time is large, so $\sigma_{i}^{z}$ has large overlap with $\mathcal{U}^{\dagger} \sigma_{i}^{z} \mathcal{U}$ and therefore will display fractional frequency oscillations. [Specifically, it follows from the construction of $\mathcal{U}$ that $\mathcal{U}=1+O(\lambda T)$, and $\lambda T \ll 1$ is the regime where the heating time is large.] We recall that the conditions for fractional frequency oscillations in the prethermalized regime are that (a) $D$ must spontaneously break the Ising symmetry $X$ up to a finite critical temperature $\tau_{c}$, and (b) the energy density with respect to $D$ of $\mathcal{U}|\psi(0)\rangle$ must correspond to a temperature $\tau<\tau_{c}$. In Fig. 1, we show the expected behavior at low temperatures $\tau$ and contrast it with the expected behavior in a system which is not a time crystal in the prethermal regime.

\section{B. Example: Periodically driven Ising spins}

Let us now consider a concrete model that realizes the behavior described above. We consider an Ising ferromagnet, with a longitudinal field applied to break the Ising symmetry explicitly, and driven at high frequency by a very strong transverse field. Thus, we take

$$
H(t)=H_{0}(t)+V
$$

(b) Non-time crystal

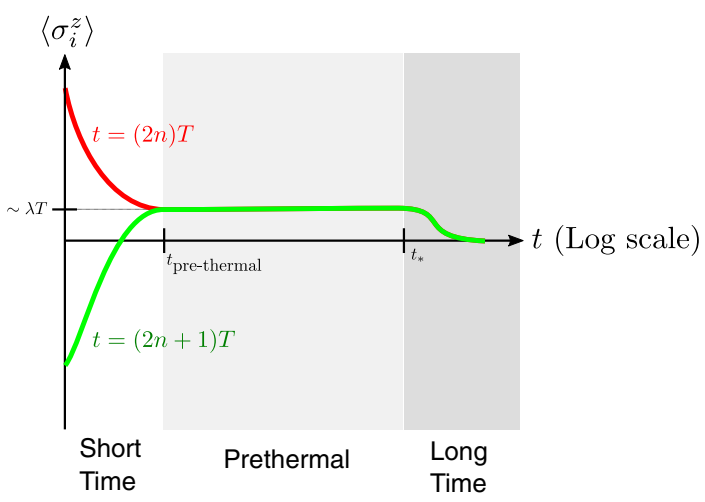

FIG. 1. The expected time dependence of $\left\langle\sigma_{i}^{z}\right\rangle$ at stroboscopic times, starting from a low-temperature state with respect to $\mathcal{U} D \mathcal{U}^{\dagger}$ (for example, for a state with all spins polarized in the $z$ direction.). Panel (a) shows the prethermal time-crystal phase, and panel (b) shows the non-time-crystal prethermal phase. 
where

$$
\begin{aligned}
H_{0}(t) & =-\sum_{i} h^{x}(t) \sigma_{i}^{x} \\
V & =-J \sum_{\langle i, j\rangle} \sigma_{i}^{z} \sigma_{j}^{z}-h^{z} \sum_{i} \sigma_{i}^{z},
\end{aligned}
$$

and we choose the driving profile such that

$$
\int_{0}^{T} h^{x}(t) d t=\frac{\pi}{2}
$$

ensuring that the "unperturbed" Floquet operator $U_{0}$ implements a $\pi$ pulse, $X=\prod_{i} \sigma_{x}^{i}$, and we can set $N=2$. (If the driving does not exactly implement a $\pi$ pulse, this is not a significant problem since we can just incorporate the difference into $V$.) This implies that $h_{x} \sim 1 / T$, and we assume that $h^{z} \lesssim J \ll 1 / T$.

Then, from the results of Sec. II (with $J$ playing the role of $\lambda$ here), we find a quasilocal Hamiltonian $D=\bar{V}+(1 / T) O\left((J T)^{2}\right)$, where

$$
\bar{V}=\frac{1}{2 T} \int_{0}^{2 T} V_{\text {int }}(t) d t
$$

In particular, in the case where the $\pi$ pulse acts instantaneously, so that

$$
h^{x}(t)=\frac{\pi}{2} \sum_{k=-\infty}^{\infty} \delta(t-k T)
$$

we find that

$$
\bar{V}=-J \sum_{\langle i, j\rangle} \sigma_{i}^{z} \sigma_{j}^{z}
$$

(this Hamiltonian is integrable, but, in general, the higherorder corrections to $D$ will destroy integrability). More generally, if the delta function is smeared out so that the $\pi$ pulse acts over a time window $\delta$, the corrections from Eq. (27) will be at most of order $\sim J \delta / T$. Therefore, so long as $\delta \ll T$, then in two dimensions or higher, the Hamiltonian $D$ will indeed spontaneously break the Ising symmetry up to some finite temperature $\tau_{c}$, and we will observe the time-crystal behavior described above.

\section{Field theory of the prethermal Floquet time-crystal state}

The universal behavior of a prethermal Floquet timecrystal state can be encapsulated in a field theory. For the sake of concreteness, we derive this theory from the model analyzed in the previous section. The Floquet operator can be written, up to nearly exponential accuracy, as

$$
U_{\mathrm{f}} \approx \mathcal{U}\left(X e^{-i D T}\right) \mathcal{U}^{\dagger}
$$

Consequently, the transition amplitude from an initial state $\left|\psi_{i}\right\rangle$ at time $t_{0}$ to a final state $\left|\psi_{f}\right\rangle$ at time $t_{0}+m T$ can be written in the following form, provided $t_{\text {pre-thermal }}<t_{0}<t_{0}+m T<t_{*}$ :

$$
\begin{aligned}
\left\langle\psi_{f}\left|\left(U_{\mathrm{f}}\right)^{m}\right| \psi_{i}\right\rangle & =\left\langle\psi_{f}\left|\mathcal{U}\left(X e^{-i D T}\right)^{m} \mathcal{U}^{\dagger}\right| \psi_{i}\right\rangle \\
& =\left\langle\tilde{\psi}_{f}\left|e^{-i D m T}\right| \tilde{\psi}_{i}\right\rangle,
\end{aligned}
$$

where $\left|\tilde{\psi}_{i}\right\rangle \equiv \mathcal{U}^{\dagger}\left|\psi_{i}\right\rangle$ and $\left|\tilde{\psi}_{f}\right\rangle \equiv X^{m} \mathcal{U}^{\dagger}\left|\psi_{f}\right\rangle$; recall that $X^{m}$ is 1 or $X$ for, respectively, $m$ even or odd.

The second line of Eq. (29) is just the transition amplitude for the quantum transverse field Ising model in $(d+1)$-dimensional spacetime, with $d \geq 2$. The model has the nearest-neighbor interaction (27) together with higher-order terms that are present in the full expression for $D$. Hence, it can be represented by the standard functional integral for the continuum limit of the Ising model:

$$
\begin{aligned}
& \left\langle\tilde{\psi}_{f}\left|e^{-i D m T}\right| \tilde{\psi}_{i}\right\rangle \\
& \quad=\int \mathcal{D} \varphi e^{i \int d^{d} x d t\left[\frac{1}{2} K\left(\partial_{t} \varphi\right)^{2}-\left(v^{2} / 2\right) K(\nabla \varphi)^{2}-U(\varphi)\right],}
\end{aligned}
$$

where $U(\varphi)$ has minima at $\varphi= \pm \varphi_{0}$ when the parameters in the Ising model place it in the ordered phase. This functional integral is only valid for wave vectors that are less than a wave-vector cutoff: $|q|<\Lambda$, where $\Lambda \ll 1 / a$ and $a$ is the spatial lattice spacing. Although the right-hand side of Eq. (30) has a continuous time variable, it is only equal to the original periodically driven problem for stroboscopic times $t=m T$ for $m \in \mathbb{Z}$. Note the left-hand side of Eq. (30) is also well defined for arbitrary times, i.e., for continuous $m$, although it, too, only corresponds to the original problem for integer $m$. Thus, the continuous-time effective field theory has a frequency cutoff $\Lambda_{\omega}$ that we are free to choose. Although the functional integral only corresponds to the original problem for stroboscopic times, the functional integral is well defined for all times. As a result of the factor of $X$ in $U_{\mathrm{f}}$, the field $\varphi$ is related to the Ising spin according to $\varphi(x, k T) \sim(-1)^{k} \sigma(x, k T)$. In other words, the field $\varphi$ in the functional integral has the interpretation of the temporally staggered magnetization density, just as, in the corresponding description of an Ising antiferromagnet, this field would be the spatially staggered magnetization. Discrete time-translation symmetry, $t \rightarrow t+T$, has the following action: $\varphi \rightarrow-\varphi$. Thus, the symmetry-breaking phase, in which $\varphi= \pm \varphi_{0}$, 
is a prethermal Floquet time crystal, in which timetranslation symmetry breaking (TTSB) occurs, as expected.

The rotated Floquet operator $\mathcal{U}^{\dagger} U_{\mathrm{f}} \mathcal{U}$ has an approximate $\mathbb{Z}_{2}$ symmetry generated by the operator $X$ since $\mathcal{U}^{\dagger} U_{\mathrm{f}} \mathcal{U} \approx$ $X e^{-i D T}$ and $[D, X]=0$. Hence, $\mathcal{U}^{\dagger} X \mathcal{U}$ commutes with the (unrotated) Floquet operator $U_{\mathrm{f}}$. It is not a microscopic symmetry in the conventional sense since $\mathcal{U}^{\dagger} X \mathcal{U}$ does not commute with the time-dependent Hamiltonian $H(t)$, except for special fine-tuned points in the Floquet timecrystal phase. However, since it commutes with the Floquet operator, it is a symmetry of the continuum-limit field theory (30). (See Ref. [45] for a discussion of Floquet time crystals in the MBL context that focuses on such symmetries, sometimes called "emergent symmetries.") Within the field theory (30), this symmetry acts according to $\varphi \rightarrow-\varphi$; i.e., it acts in precisely the same way as time translation by a single period. Again, this is analogous to the case of an Ising antiferromagnet but with the time translation taking the place of spatial translation. Thus, it is possible to view the symmetry-breaking pattern as $\mathbb{Z}_{\text {TTS }} \times \mathbb{Z}_{2} \rightarrow \mathbb{Z}$. The unbroken $\mathbb{Z}$ symmetry is generated by the combination of time translation by one period and the action of $\mathcal{U}^{\dagger} X \mathcal{U}$.

However, there is an important difference between a Floquet time crystal and an Ising antiferromagnet. In the latter case, it is possible to explicitly break the Ising symmetry without breaking translational symmetry (e.g., with a uniform longitudinal magnetic field) and vice versa (e.g., with a spatially oscillating exchange coupling). In a Floquet time crystal, this is not possible because there is always a $\mathbb{Z}_{2}$ symmetry $\mathcal{U}^{\dagger} X \mathcal{U}$ regardless of what small perturbation (compared to the drive frequency) is added to the Hamiltonian. The only way to explicitly prevent the system from having a $\mathbb{Z}_{2}$ symmetry is to explicitly break the time-translation symmetry. Suppose the Floquet operator is $\mathcal{U} X e^{-i D T} \mathcal{U}^{\dagger}$. When a weak perturbation with period $2 T$ is added, the Floquet operator can be written in the approximate form $\mathcal{U}^{\prime} e^{-2 i(D+Y) T}\left(\mathcal{U}^{\prime}\right)^{\dagger}$, where $Y$ is due to the doubled-period weak perturbation, but it is not possible to guarantee that $[X, Y]=0$. Thus, there is a symmetry generated by an operator of the form $\mathcal{U}^{\dagger} X \mathcal{U}$ only if time-translation symmetry is present-i.e., it is a consequence of timetranslation symmetry and prethermalization.

This functional integral is computed with boundary conditions on $\varphi$ at $t=t_{0}$ and $t_{0}+m T$. Time-ordered correlation functions can be computed by inserting operators between the factors of $U_{\mathrm{f}}$. However, if we are interested in equal-time correlation functions (at stroboscopic times $t=k T$ ),

$$
\begin{aligned}
& \langle\psi|\hat{O}(x, k T) \hat{O}(0, k T)| \psi\rangle \\
& \quad \equiv\left\langle\psi\left|\left(U_{\mathrm{f}}\right)^{-k} \hat{O}(x, 0) \hat{O}(0,0)\left(U_{\mathrm{f}}\right)^{k}\right| \psi\right\rangle
\end{aligned}
$$

then we can make use of the fact that the system rapidly prethermalizes to replace $\left(U_{\mathrm{f}}\right)^{k}|\psi\rangle$ by a thermal state:

$$
\begin{aligned}
& \left\langle\psi\left|\left(U_{\mathrm{f}}\right)^{-k} \hat{O}(x, 0) \hat{O}(0,0)\left(U_{\mathrm{f}}\right)^{k}\right| \psi\right\rangle \\
& \quad=\operatorname{tr}\left(e^{-\beta D} \hat{O}(x) \hat{O}(0)\right)
\end{aligned}
$$

where $\beta$ is determined by $\operatorname{tr}\left(e^{-\beta D} D\right)=\langle\psi|D| \psi\rangle$. The latter has an imaginary-time functional integral representation:

$$
\begin{aligned}
& \operatorname{tr}\left(e^{-\beta D} \hat{O}(x) \hat{O}(0)\right) \\
& \quad=\int \mathcal{D} \varphi e^{-\int d^{d} x d \tau\left[\frac{1}{2} K\left(\partial_{\tau} \varphi\right)^{2}+\left(v^{2} / 2\right) K(\nabla \varphi)^{2}+U(\varphi)\right] .}
\end{aligned}
$$

This equation expresses equal-time correlation functions in a prethermal Floquet time crystal in terms of the standard imaginary-time functional integral for the Ising model but with the understanding that the field $\varphi$ in the functional integral is related to the Ising spins in the manner noted above.

In order to compute unequal-time correlation functions, it is convenient to use the Schwinger-Keldysh formalism [81,82] (see Ref. [83] for a modern review). This can be done by following the logic that led from the first line of Eq. (29) to the second and thence to Eq. (30). This will be presented in detail elsewhere [84].

We close this subsection by noting that the advantage of the field theory formulation of a prethermal Floquet time crystal is the salience of the similarity with the equilibrium Ising model; for instance, it is clear that the transition out of the Floquet time crystal (e.g., as a function of the energy of the initial state) in the prethermal regime is an ordinary Ising phase transition. The disadvantage is that it is difficult to connect it to measurable properties in a quantitative way because the field $\varphi$ has a complicated relationship with the microscopic degrees of freedom.

\section{Relation to formal definitions of time crystals}

In the above discussion, we have implicitly been adopting an "operational" definition of time crystal: It is a system in which, for physically reasonable initial states, the system displays oscillations at a frequency other than the drive frequency forever (or at least, in the prethermal case, for a nearly exponentially long time). This is a perfectly reasonable definition of time crystal, but it has the disadvantage of obscuring the analogies with spontaneous breaking of other symmetries, which tends not to be defined in this way. (Although in fact it could be; for example, an "operational" definition of spontaneously broken Ising symmetry would be a system in which the symmetrybreaking order parameter does not decay with time for physically reasonable initial states [85].) For this reason, in Ref. [43], we introduced a formal definition of timetranslation symmetry breaking in MBL systems in terms 
of eigenstates (two equivalent formulations, which we called TTSB-1 and TTSB-2).

The definitions TTSB-1 and TTSB-2 of Ref. [43] are natural generalizations of the notion of "eigenstate order" used to define spontaneous breaking of other symmetries in MBL $[85,86]$. On the other hand, they (like the notion of eigenstate order, in general) are not really appropriate outside of the MBL context. In this subsection, we review the usual formal definitions of spontaneous symmetry breaking in equilibrium. Then, we show how they can be extended in a natural way to timetranslation symmetries, as well as how these extended versions are satisfied by the prethermal Floquet time crystals constructed above.

Let us first forget about time-translation symmetry and consider a time-independent Hamiltonian $H$ with an Ising symmetry generated by $X$. Let $\rho$ be a steady state of the Hamiltonian; that is, it is invariant under the time evolution generated by $H$. (Here, we work in the thermodynamic limit, so by $\rho$, we really mean a function that maps local observables to their expectation values; that is, we define a state in the $C^{*}$-algebra sense [87].) Generically, we expect $\rho$ to be essentially a thermal state. If the symmetry is spontaneously broken, then $\rho$ can obey the cluster decomposition (i.e., its correlations can be short ranged), or it can be invariant under the symmetry $X$, but not both. In other words, any state invariant under the symmetry decomposes as $\rho=\frac{1}{2}\left(\rho_{\uparrow}+\rho_{\downarrow}\right)$, where $\rho_{\uparrow}$ and $\rho_{\downarrow}$ have opposite values of the Ising order parameter and are mapped into each other under $X$. Thus, a formal definition of spontaneously broken Ising symmetry can be given as follows. We call a symmetry-invariant steady state $\rho$ an extremal symmetryrespecting state if states $\rho_{1}$ and $\rho_{2}$ do not exist, such that $\rho=p \rho_{1}+(1-p) \rho_{1}$ for some $p \in(0,1)$, where $\rho_{1}$ and $\rho_{2}$ are symmetry-invariant steady states. We say the Ising symmetry is spontaneously broken if extremal symmetryinvariant steady states do not satisfy the cluster decomposition. Similar statements can be made for Floquet systems, where by "steady state" we now mean a state that returns to itself after one time cycle.

We can now state the natural generalization to timetranslation symmetry. For time-translation symmetry, "symmetry-invariant" and "steady state" actually mean the same thing. So we say that time-translation symmetry is spontaneously broken if extremal steady states do not satisfy the cluster decomposition. This is similar to our definition TTSB-2 from Ref. [43] (but not exactly the same since TTSB-2 was expressed in terms of eigenstates rather than extremal steady states in an infinite system), so we call it TTSB- $2^{\prime}$. We note that TTSB-2 ${ }^{\prime}$ implies that any shortrange correlated state $\rho$, i.e., a state $\rho$ that satisfies the cluster decomposition, must not be an extremal steady state. Nonextremal states never satisfy the cluster decomposition; thus, we conclude that short-range correlated states must not be steady states at all, so they cannot simply return to themselves after one time cycle. (This is similar to, but again not identical to, TTSB-1 in Ref. [43].)

We note that, for clean systems, the only steady state of the Floquet operator $U_{\mathrm{f}}$ is believed to be the infinite temperature state [46-48], which always obeys the cluster property; hence, time-translation symmetry is not broken spontaneously. This does not contradict our previous results since we already saw that time-translation symmetry is only spontaneously broken in the prethermal regime, not at infinitely long times. Instead, we should examine the steady states of the approximate Floquet operator $\tilde{U}_{\mathrm{f}}$, which describes the dynamics in the prethermal regime. We recall that, after a unitary change of basis, $\tilde{U}_{\mathrm{f}}=X e^{-i D T}$, where $D$ commutes with $X$ and spontaneously breaks the Ising symmetry generated by $X$ (for temperatures $\tau<\tau_{c}$ ). Hence, $\tilde{U}_{\mathrm{f}}^{2}=e^{-2 i D T}$. Any steady state $\rho$ of $\tilde{U}_{\mathrm{f}}$ must be a steady state of $\tilde{U}_{\mathrm{f}}^{2}$, which implies (if its energy density corresponds to a temperature $\tau<\tau_{c}$ ) that it must be of the form $\rho=t \rho_{\mathrm{SB}}+(1-t) X \rho_{\mathrm{SB}} X$, where $\rho_{\mathrm{SB}}$ is an Ising symmetry-breaking state of temperature $\tau$ for the Hamiltonian $D$. Hence, we see (since $\rho_{\mathrm{SB}}$ is invariant under $\left.e^{-i D T}\right)$ that $\tilde{U}_{\mathrm{f}} \rho \tilde{U}_{\mathrm{f}}^{\dagger}=t X \rho_{\mathrm{SB}} X+(1-t) \rho_{\mathrm{SB}}$. So if $\rho$ is a steady state of $\tilde{U}_{\mathrm{f}}$ and not just $\tilde{U}_{\mathrm{f}}^{2}$, we must have $t=1 / 2$. But then the state $\rho$ clearly violates the cluster property. Hence, time translation is spontaneously broken.

\section{SPONTANEOUSLY BROKEN CONTINUOUS TIME-TRANSLATION SYMMETRY IN THE PRETHERMAL REGIME}

\section{A. Basic picture}

The prethermalized Floquet time crystals discussed above have a natural analog in undriven systems with continuous time-translation symmetry. Suppose we have a time-independent Hamiltonian

$$
H=-u L+V,
$$

where the eigenvalues of $L$ are integers; in other words, for time $T=2 \pi / u$, the condition $e^{i n u L T}=1$ holds for all $n \in \mathbb{Z}$. We also assume that $L$ is a sum of local terms of local strength $O(1)$, and $V$ is a local Hamiltonian of local strength $\lambda \ll u$. Then, by Theorem 3.1 of Ref. [74], restated in Theorem 2 in Sec. II), there exists a local unitary $\mathcal{U}$ such that $\mathcal{U} H \mathcal{U}^{\dagger}=-u L+D+\hat{V}$, where $[D, L]=0$ and the local strength of $\hat{V}$ is $\sim \lambda e^{-O\left([\log \lambda T]^{3} /[\lambda T]\right)}$. As noted in Theorem 2 in Sec. II, the first term in the explicit iterative construction of $D$ in Ref. [74] is $D=\langle V\rangle+(1 / T) O(\lambda T)^{2}$, where

$$
\langle V\rangle \equiv \frac{1}{2 \pi} \int_{0}^{2 \pi} d \theta e^{i L \theta} V e^{-i L \theta} .
$$

As a result of this theorem, such a system has an approximate $\mathrm{U}(1)$ symmetry generated by $\mathcal{U}^{\dagger} L \mathcal{U}$ that is 
explicitly broken only by nearly exponentially small terms. Consequently, $\mathcal{U}^{\dagger} L \mathcal{U}$ is conserved by the dynamics of $H$ for times $t \ll t_{*}=e^{O\left([-\log \lambda T]^{3} /[\lambda T]\right)}$. We will call the Hamiltonian $-u L+D$ the "prethermal" Hamiltonian since it governs the dynamics of the system for times that are short compared to $t_{*}$. We assume that we have added a constant to the Hamiltonian such that $L$ is positive definite; this will allow us to abuse terminology a little by referring to the expectation value of $L$ as the "particle number," in order to make analogies with well-known properties of Bose gases, in which the generator of the $\mathrm{U}(1)$ symmetry is the particle number operator. In this vein, we call $u$ the electric potential, in analogy with (negatively) charged superfluids.

We further suppose that $D$ is neither integrable nor many-body localized, so the dynamics of $D$ will cause an arbitrary initial state $\left|\psi_{0}\right\rangle$ with nonzero energy density and nonzero $\left\langle\psi_{0}|L| \psi_{0}\right\rangle$ to rapidly thermalize on some short (compared to $t_{*}$ ) time scale $t_{\text {pre-thermal }} \sim \lambda^{-1}$. The resulting thermalized state can be characterized by the expectation values of $D$ and $L$, both of which will be the same as in the initial state since energy and particle number are conserved. Equivalently, the thermalized state can be characterized by its temperature $\beta$ (defined with respect to $D$ ) and effective chemical potential $\mu$. In other words, all local correlation functions of local operators can be computed with respect to the density matrix $\rho=e^{-\beta(D-\mu L)}$. The chemical potential $\mu$ has been introduced to enforce the condition $\operatorname{tr}(\rho L)=\left\langle\psi_{0}|L| \psi_{0}\right\rangle$.

Now, suppose that we choose $V$ such that $D$ spontaneously breaks the $U(1)$ symmetry in some range of temperature $1 / \beta$ and chemical potential $\mu$. Suppose, further, that we prepare the system in a short-range correlated initial state $\left|\psi_{0}\right\rangle$ such that the energy density (and, hence, its temperature) is sufficiently low, and the number density sufficiently high, so that the corresponding thermalized state spontaneously breaks the U(1) symmetry generated by $L$. Then, the preceding statement must be slightly revised: All local correlation functions of local operators can be computed with respect to the density matrix $\rho=$ $e^{-\beta(D-\mu L-\epsilon X)}$ for some $X$ satisfying $[X, L] \neq 0$. The limit $\epsilon \rightarrow 0$ is taken after the thermodynamic limit is taken; the direction of the infinitesimal symmetry-breaking field $X$ is determined by the initial state. To avoid clutter, we will not write $\epsilon X$ explicitly in the next paragraph, but it is understood.

Consider an operator $\Phi$ that satisfies $[L, \Phi]=\Phi$. (For example, if we interpret $L$ as the particle number, we can take $\Phi$ to be the particle creation operator.) Its expectation value at time $t$ is given by

$$
\begin{aligned}
& \left\langle\psi_{0}\left|e^{-i(-u L+D) t} \Phi e^{i(-u L+D) t}\right| \psi_{0}\right\rangle \\
& \quad=\operatorname{tr}\left(\left[e^{-i(-u L+D) t} \Phi e^{i(-u L+D) t}\right] e^{-\beta(D-\mu L)}\right) \\
& =e^{i(\mu-u) t} \operatorname{tr}\left(\left[e^{-i(-\mu L+D) t} \Phi e^{i(-\mu L+D) t}\right] e^{-\beta(D-\mu L)}\right) .
\end{aligned}
$$

According to the discussion in Appendix B, which makes use of the result of Watanabe and Oshikawa [42], the trace on the right-hand side of the second equality must be independent of time. Hence, so long as $\operatorname{Tr}\left(\Phi e^{-\beta(D-\mu L)}\right) \neq 0$ (which we assume to be true for some order parameter $\Phi$ in the symmetry-breaking phase), we find that the expectation value of $\Phi$ oscillates with frequency given by the "effective electrochemical potential" $\mu-u$ due to the winding of the phase of $\Phi$.

If the dynamics were exactly governed by $-u L+D$, then the system would oscillate with period $2 \pi /(u-\mu)$ forever. As it is, these oscillations will be observed until the exponentially late time $t_{*}$. At infinitely long times, the system approaches a thermal state of the full Hamiltonian $-u L+D+\hat{V}$. Since $\hat{V}$ is small, this is approximately the same as a thermal state of $-u L+D$. However, because $\hat{V}$ is not exactly zero, the particle number is not conserved, and in equilibrium, the system chooses the particle number that minimizes its free energy, which corresponds to the electrochemical potential being zero, $\mu-u=0$. Since this corresponds to zero frequency of oscillations, it follows that no oscillations are observed at infinite time.

The above discussion is essentially the logic that was discussed in Refs. [41,42,88], where it was pointed out that a superfluid at nonzero chemical potential is a time crystal as a result of the well-known time dependence of the order parameter [89]. However, there is an important difference: The $\mathrm{U}(1)$ symmetry is not a symmetry of the Hamiltonian of the problem; therefore, it does not require fine-tuning but, instead, emerges in the $u \rightarrow \infty$ limit, thereby evading the criticism $[41,42,90-92]$ that the phase winds in the ground state only if the U(1) symmetry is exact.

\section{B. Example: $X Y$ ferromagnet in a large perpendicular field}

Consider the concrete example of a spin- $1 / 2$ system in three spatial dimensions, with Hamiltonian

$$
\begin{aligned}
H= & -h^{z} \sum_{i} S_{i}^{z}-h^{x} \sum_{i} S_{i}^{x} \\
& -\sum_{i, j}\left[J_{i j}^{x} S_{i}^{x} S_{j}^{x}+J_{i j}^{y} S_{i}^{y} S_{j}^{y}+J_{i j}^{z} S_{i}^{z} S_{j}^{z}\right] .
\end{aligned}
$$

We take $L=S^{z} \equiv \sum_{i} S_{i}^{z}$, and the longitudinal magnetic field $h^{z}$ plays the role of $u$ in the preceding section. We take $J_{i j}$ and $J_{i j}^{z}$ to vanish except for nearest neighbors, for which $J_{i j}^{x}=J+\delta J, J_{i j}^{y}=J_{y}+\delta J$, and $J_{i j}^{z}=J^{z}$. (We do not assume $\delta J \ll J$.) The local scale of $V$ is given by $\lambda=\max \left(J+\delta J, h^{x}\right)$, so the condition $\lambda \ll T^{-1} \sim h^{z}$ is satisfied if $J+\delta J, h^{x} \ll h^{z}$. In this case, $D$ is (to first order) the Hamiltonian of an $X Y$ ferromagnet: 


$$
D=-\sum_{\langle i, j\rangle}\left[J\left(S_{i}^{x} S_{j}^{x}+S_{i}^{y} S_{j}^{y}\right)+J^{z} S_{i}^{z} S_{j}^{z}\right]+\frac{1}{T} O\left(\lambda / h^{z}\right)^{2} .
$$

Then, starting from a short-range correlated state with appropriate values of energy and $\left\langle S^{z}\right\rangle$, we expect that time evolution governed by $D$ causes the system to "prethermalize" into a symmetry-breaking state with some value of the order parameter $\left\langle S_{i}^{+}\right\rangle=n_{0} e^{i \phi}$. According to the preceding discussion, the order parameter will then rotate in time with angular frequency $\omega=\mu-h^{z}$ (where $\mu \lesssim \lambda$ is determined by the initial value of $\left\langle S^{z}\right\rangle$ ) for times that are short compared to the thermalization time $t_{*}$.

Note, however, that we have assumed that the system is completely isolated. If the system is not isolated, then the periodic rotation of the order parameter will cause the system to emit radiation, and this radiation will cause the system to decay to its true ground state $[37,93]$.

\section{Field theory of prethermal continuous-TTSB time crystal}

For simplicity, we only give the imaginary-time field theory for equal-time correlation functions deep within the prethermal regime; the Schwinger-Keldysh functional integral for unequal-time correlation functions, with nearly exponentially small thermalization effects taken into account, will be discussed elsewhere [84]. Introducing the field $\phi \sim\left(S_{x}+i S_{y}\right) e^{i(\mu-u) t}$, we apply Eq. (36) to the $X Y$ ferromagnet of the previous section, thereby obtaining the effective action:

$S_{\mathrm{eff}}=\int d^{d} x d \tau\left[\phi^{*} \partial_{\tau} \phi-\mu \boldsymbol{\phi}^{*} \phi+g\left(\boldsymbol{\phi}^{*} \phi\right)^{2}+\cdots\right]$.

The ellipsis represents higher-order terms. The U(1) symmetry generated by $S^{z}$ acts according to $\phi \rightarrow e^{i \theta} \phi$. Time-translation symmetry acts according to $\phi(t) \rightarrow$ $e^{i(\mu-u) a} \phi(t+a)$ for any $a$. Thus, when $\phi$ develops an expectation value, both symmetries are broken, and a combination of them is preserved according to the symmetry-breaking pattern $\mathbb{R}_{\text {TTS }} \times \mathrm{U}(1) \rightarrow \mathbb{R}$, where the unbroken $\mathbb{R}$ is generated by a gauge transformation by $\theta$ and a time translation $t \rightarrow t+(\theta / \mu-u)$.

From the mathematical equivalence of Eq. (39) to the effective field theory of a neutral superfluid, we see that (1) in $2 \mathrm{D}$, there is a quasi-long-range-ordered phase-an "algebraic time crystal"- for initial-state energies below a Kosterlitz-Thouless transition; (2) the TTSB phase transition in $3 \mathrm{D}$ is in the ordinary $X Y$ universality class in $3 \mathrm{D}$; (3) the 3D time-crystal phase has Goldstone boson excitations. If we write $\phi(x, t)=\sqrt{((\mu / 2 g)+\delta \rho(x, t))} e^{i \theta(x, t)}$ and integrate out the gapped field $\delta \rho(x, t)$, then the effective action for the gapless Goldstone boson $\theta(x, t)$ is of the form discussed in Ref. [91].

\section{PRETHERMALIZED FLOQUET TOPOLOGICAL PHASES}

We can also apply our general results of Sec. II to Floquet SPT and SET topological phases, even those which do not exist in stationary systems. (We will henceforth use the abbreviation SxT to refer to either SPT or SET phases.)

As was argued in Refs. [31,32], any such phase protected by symmetry $G$ is analogous to a topological phase of a stationary system protected by symmetry $\mathbb{Z} \rtimes G$, where the extra $\mathbb{Z}$ corresponds to the time-translation symmetry. Here, the product is semidirect for anti-unitary symmetries and direct for unitary symmetries. For simplicity, here we consider only unitary symmetries. Similar arguments can be made for anti-unitary symmetries.

We consider the class of phases that can still be realized when the $\mathbb{Z}$ is refined to $\mathbb{Z}_{N}$. In other words, the analogous stationary phase can be protected by a unitary representation $W(\tilde{g})$ of the group $\tilde{G}=\mathbb{Z}_{N} \times G$. Then, in applying the general result of Sec. II, we choose $H_{0}(t)$ such that its time evolution over one time cycle is equal to $X \equiv W(\mathbb{T})$, where $\mathbb{T}$ is the generator of $\mathbb{Z}_{N}$. Then, it follows that, for a generic perturbation $V$ of small enough local strength $\lambda$, there exists a local unitary rotation $\mathcal{U}$ (commuting with all the symmetries of $U_{\mathrm{f}}$ ) such that $U_{\mathrm{f}} \approx \tilde{U}_{\mathrm{f}}$, where $\tilde{U}_{\mathrm{f}}=\mathcal{U} X e^{-i D T} \mathcal{U}^{\dagger}$, $D$ is a quasilocal Hamiltonian that commutes with $X$, and $\tilde{U}_{\mathrm{f}}$ describes the dynamics well until the almost exponentially large heating time $t_{*}$.

Now, let us additionally assume (since we want to construct a Floquet-SxT protected by the symmetry $G$, plus time translation) that the Floquet operator $U_{\mathrm{f}}$ is chosen such that it has the symmetry $G$. Specifically, this means that it is generated by a periodic time evolution $H(t)$ such that, for all $g \in G, W(g) H(t) W(g)^{-1}$. By inspection of the explicit construction for $\mathcal{U}$ and $D$ (see Appendix A), it is easy to see that, in this case, $\mathcal{U}$ is a symmetry-respecting local unitary with respect to $W(g)$, and $D$ commutes with $W(g)$. In other words, the rotation by $\mathcal{U}$ preserves the existing symmetry $G$ and also reveals a new $\mathbb{Z}_{N}$ symmetry generated by $X$ (which was "hidden" in the original frame).

Therefore, we can choose $D$ to be a Hamiltonian whose ground state is in the stationary SxT phase protected by $\mathbb{Z}_{N} \times G$. It follows (by the same arguments discussed in Ref. [31] for the MBL case) that the ground state $D$ will display the desired Floquet-SxT order under the time evolution generated by $\mathcal{U}^{\dagger} U_{\mathrm{f}} \mathcal{U}=X e^{-i D T}$. Furthermore, since Floquet-SxT order is invariant under symmetryrespecting local unitaries, the ground state of $\mathcal{U D U}^{\dagger}$ will display the desired Floquet-SxT order under $U_{\mathrm{f}}$.

Note, however, that topological order, in contrast to symmetry-breaking order, does not exist at nonzero temperature (in clean systems, for spatial dimensions $d<4$ ). 
Thus, for initial-state mean energies $\langle D\rangle$ that correspond to temperatures $\beta^{-1}$ satisfying $0<\beta^{-1} \ll \Delta$, where $\Delta$ is the bulk energy gap, the system will exhibit exponentially small corrections $\sim e^{-\beta \Delta}$ to the quantized values that would be observed in the ground state. This is no worse than the situation in thermal equilibrium, where, for instance, the Hall conductance is not precisely quantized in experiments but has small corrections $\sim e^{-\beta \Delta}$. However, preparing such an initial state will be more involved than for a simple symmetry-breaking phase. For this reason, it is more satisfactory to envision cooling the system by coupling to a thermal bath, as discussed in Sec. VI, which is analogous to how topological phases are observed in thermal equilibrium experiments - by refrigeration.

\section{OPEN SYSTEMS}

So far, we have considered only isolated systems. In practice, of course, some coupling to the environment will always be present. One can also consider the effect of classical noise-for example, some time-dependent randomness in the parameters of the drive-so that successive time steps will not implement exactly the same time evolution. The Floquet-MBL time crystals of Ref. [43] are not expected to remain robust in such setups because MBL will be destroyed. Since some amount of coupling to the environment is inevitable in realistic setups, this limits the time scales over which one could expect to observe Floquet-MBL time crystals experimentally.

However, the situation could be quite different for the prethermal time crystals of this work. A complete treatment is beyond the scope of the present work, so in this section, we confine ourselves to stating one very interesting hypothesis: Floquet case time crystals can actually be stabilized in open systems so that the oscillations actually continue forever for any initial state (in contrast to the case of isolated systems, in which, as discussed previously, the oscillations continue only up to some very long time and only for some initial states). We will not attempt to establish this more rigorously but simply discuss a plausible scenario by which this would occur. The idea, as depicted in Fig. 2, is that the heating due to the periodic driving, as well as classical noise sources and other stray couplings to an environment, can be counteracted by cooling from a coupling to a sufficiently cold thermal bath. Provided that the resulting steady state has sufficiently low "energy," we argue that oscillations at a fraction of the drive frequency will be observed in this steady state. Here, energy means the expectation value of the effective Hamiltonian $D$, which describes the dynamics in the prethermal regime. We discuss this hypothesis further, and show that it indeed implies periodic oscillations, in Appendixes C and D. We also note that this argument does not apply to the continuous-time time crystals of Sec. IV since, in that case, low energy is not a sufficient condition to observe

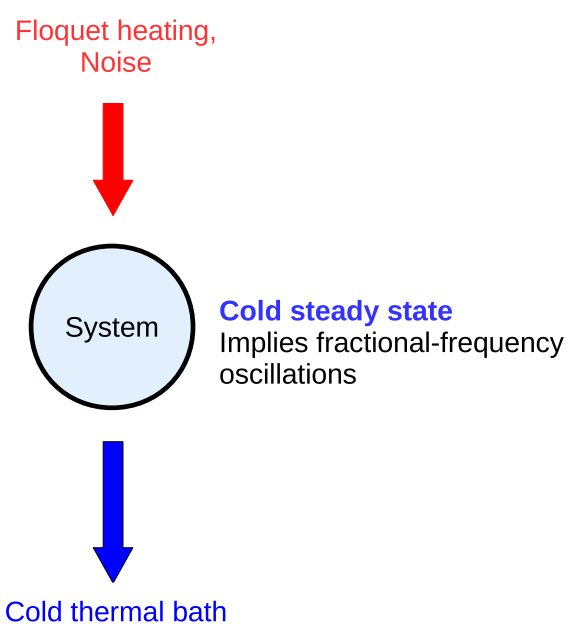

FIG. 2. So long as the energy inflow due to noise and periodic driving is balanced by the outflow to a cold thermal bath, giving a low-energy steady state, oscillations at a fraction of the drive frequency will be observed.

oscillations even in an isolated system; there is also a dependence on the chemical potential $\mu$.

\section{DISCUSSION}

In this paper, we described how phases protected by time-translation symmetry can be observed in the prethermal regime of driven and undriven quantum systems. This greatly increases the set of experimental systems in which such phases can be observed since, as opposed to previous proposals, we do not require many-body localization to robustly prevent the system from heating to infinite temperature. While many-body localization has been observed in experiments [94-96], the ideas put forward in this paper significantly reduce experimental requirements, as strong disorder is not required.

Our Theorem 1 implies that the time-translationprotected behavior (for example, the fractional-frequency oscillations in the Floquet time crystal) can be observed to nearly exponentially late times, provided that the drive frequency is sufficiently high. However, the rigorous bound given in the theorem-which requires a drive frequency about $10^{3}$ times larger than the local couplings in the timedependent Hamiltonian-may not be tight. Therefore, it would be interesting to check numerically whether (in the Floquet time-crystal case, say) long-lived oscillations are observed in systems with drive frequency only moderately larger than the local couplings. This may be challenging in small systems, in which there is not a large separation of energy scales between the local coupling strength and the width of the many-body spectrum (which the frequency should certainly not exceed). In one-dimensional systems, oscillations will not be observed to exponentially long (in the drive frequency) times but will have a finite correlation time for any nonzero energy density initial 
state. However, there will be a universal quantum critical regime in which the correlation time will be the inverse effective temperature.

Although naive application of Theorem 1 suggests that the ideal situation is the one in which the drive frequency becomes infinitely large, in practice, very high-frequency driving will tend to excite high-energy modes that were ignored in constructing the model lattice Hamiltonian. For example, if the model Hamiltonian describes electrons moving in a periodic potential in the tight-binding approximation, high-frequency driving would excite higher orbitals that were excluded. Thus, the driving frequency $\Omega$ needs to be much greater than the local energy scales of the degrees of freedom included in the model Hamiltonian (except for one particular coupling, as discussed in Sec. III) but also much less than the local energy scales of the degrees of freedom not included. (One cannot simply include all degrees of freedom in the model Hamiltonian, because then the norm of local terms would be unbounded, and Theorem 1 would not apply.)

In the case of undriven systems, we have shown that continuous time-translation symmetry breaking can similarly occur on nearly exponentially long time intervals even without any fine-tuning of the Hamiltonian, provided that there is a large separation of scales in the Hamiltonian. We show how, in certain cases, this can be described in terms of approximate Goldstone bosons associated with the spontaneously broken time-translation symmetry.

Our analysis relied on the construction of hidden approximate symmetries that are present in a prethermal regime. The analogous symmetries in MBL systems, where they are exact, were elucidated in the interesting work of von Keyserlingk et al. [45]. In the time-translation protected phases discussed here, the symmetry generated by the operator $\mathcal{U}^{\dagger} X \mathcal{U}$ is enslaved to time-translation symmetry since, in the absence of fine-tuning, such a symmetry exists only if time-translation symmetry is present. (In other words, if we add fields to the Hamiltonian that are periodic with period $n T$ and not period $T$, then the hidden symmetry no longer exists.) Moreover, this symmetry is broken if and only if time-translation symmetry is broken. (Similar statements hold in the MBL case [45].) In the Floquet timecrystal case, the hidden symmetry generated by $\mathcal{U}^{\dagger} X \mathcal{U}$ acts on the order parameter at stroboscopic times in the same way as time translation by $T$ (a single period of the drive), and therefore, it does not constrain correlation functions any more than they already are constrained by time-translation symmetry. The same observation holds for the approximate symmetry generated by $L_{z}$ in the undriven case.

However, there are systems in which time-crystal behavior actually does "piggyback" off another broken symmetry. This does require fine-tuning since it is necessary to ensure that the system possesses the "primary" symmetry, but such tuning may be physically natural [e.g., helium atoms have a very long lifetime, leading to a U(1) symmetry]. The broken symmetry allows a manybody system to effectively become a few-body system. Thus, time-crystal behavior can occur in such systems for the same reason that oscillations can persist in few-body systems. Oscillating Bose condensates (e.g., the ac Josephson effect and the model of Ref. [97]) can thus be viewed as fine-tuned time crystals. They are not stable to arbitrary time-translation symmetry-respecting perturbations; a perturbation that breaks the "primary" symmetry will cause the oscillations to decay. Indeed, most few-body systems are actually many-body systems in which a spontaneously broken symmetry approximately decouples a few degrees of freedom. A pendulum is a system of $10^{23}$ atoms that can be treated as a single rigid body due to spontaneously broken spatial translational symmetry: Its oscillations owe their persistence to this broken symmetry, which decouples the center-of-mass position from the other degrees of freedom.

With the need for MBL obviated by prethermalization, we have opened up the possibility of time-translation protected phases in open systems, in which MBL is impossible [64-72]. In fact, since the results of Appendix D show that TTSB can occur in nonthermal states, it is possible for the coupling to a cold bath to counteract the heating effect that would otherwise bring an end to the prethermal state at time $t_{*}$. This raises the possibility of time-translation protected phases that survive to infinite times in nonequilibrium steady states; the construction of such states is an interesting avenue for future work.

\section{ACKNOWLEDGMENTS}

We thank D. E. Liu and T. Karzig for helpful discussions. We thank W. W. Ho for pointing out a typo in a previous version of the manuscript. D.E. acknowledges support from the Microsoft Corporation. Part of this work was completed at the Aspen Center for Physics, which is supported by National Science Foundation Grant No. PHY-1066293.

Note added.-Recently, two experimental papers (J. Zhang et al., arXiv:1609.08684 and S. Choi et al., arXiv:1610.08057) have appeared with evidence consistent with the observation of a Floquet time crystal. We note that the paper by J. Zhang et al. implements disorder by addressing each ion sequentially. A prethermal version of this experiment would not need disorder, thereby sidestepping this bottleneck standing in the way of experiments on larger systems. The paper by Choi et al. studies a system that is unlikely to be many-body localized and therefore occurs during a slow approach to equilibrium. This is unlikely to correspond to a prethermal regime, but the approximate short-time form of the time evolution entailed in our Theorem 1 might still be relevant to understanding the results. 


\section{APPENDIX A: RIGOROUS PROOF OF PRETHERMALIZATION RESULTS}

\section{Definition of the norm}

Let us suppose, for the sake of concreteness, that we have a spin system with a local time-dependent Hamiltonian of the form

$$
\begin{aligned}
H(t) & =\sum_{i, j} J_{i, j}^{\alpha \beta}(t) S_{i}^{\alpha} S_{j}^{\beta}+\sum_{i, j, k} K_{i, j, k}^{\alpha \beta \gamma}(t) S_{i}^{\alpha} S_{j}^{\beta} S_{k}^{\gamma}+\cdots \\
& =\sum_{p} \sum_{p \text {-tuples }} A_{i_{1}, \ldots, i_{p}} .
\end{aligned}
$$

Here, $\alpha=x, y, z$ are the components of the spins, and $i, j, k$ are lattice sites. In the first line, we have explicitly written the two-site and three-site terms; the ellipsis represents terms up to $n$-site terms, for some finite $n$. It is assumed that these interactions have a finite range $r \geq n$ such that all of the sites in a $k$-site term are within distance $r$. In the second line, we have reexpressed the Hamiltonian in a more generic form in terms of $p$-site terms $A_{i_{1}, \ldots, i_{p}}$ with $i_{1} \neq \ldots \neq i_{p}$. To avoid clutter, we have not explicitly denoted the $t$ dependence of $A_{i_{1}, \ldots, i_{p}}$. We define the local instantaneous norm $\left\|A_{i_{1}, \ldots, i_{p}}\right\|_{n}$ according to

$$
\left\|A_{i_{1}, \ldots, i_{p}}\right\|_{n}^{\text {inst }} \equiv e^{p \kappa_{n}}\left\|A_{i_{1}, \ldots, i_{p}}\right\|
$$

where $\left\|A_{i_{1}, \ldots, i_{p}}\right\|$ is the operator norm of $A_{i_{1}, \ldots, i_{p}}$ at a given instant of time $t$ and

$$
\kappa_{n} \equiv \kappa_{1} /[1+\ln n] .
$$

We make this choice of $n$ dependence of $\kappa_{n}$, following Ref. [74], for reasons that will be clear later. We then average the instantaneous norm over one cycle of the drive:

$$
\left\|A_{i_{1}, \ldots, i_{p}}\right\|_{n} \equiv \frac{1}{T} \int_{0}^{T} d t\left\|A_{i_{1}, \ldots, i_{p}}\right\|_{n}^{\text {inst }} .
$$

It is only in this step that we differ from Abanin et al. [74], who consider the supremum over $t$ rather than the average. In analyzing the Floquet operator, i.e., the evolution due to $H$ at stroboscopic times, it is the total effect of $H$, which is determined by its integral over a cycle, that concerns us. Error terms that act over a very short time, even if they are relatively strong, have little effect on the Floquet operator so long as their norm, as defined above, is small. Finally, we define the global time-averaged norm of the Hamiltonian $H$ :

$$
\|H\|_{n} \equiv \sup _{j} \sum_{p} \sum_{p \text {-tuples }}\left[\sum_{k} \delta_{j, i_{k}}\right]\left\|A_{i_{1}, \ldots, i_{p}}\right\|_{n} .
$$

The term in square brackets restricts the sum to $p$-tuples that contain the site $j$.

\section{More technical statement of Theorem 1}

Theorem 1 stated above will follow from the slightly more technical formulation below. For notational simplicity, we work in units with $T=1$.

Theorem 1'. Consider a periodically driven system with a Floquet operator:

$$
U_{f}=\mathcal{T} \exp \left(-i \int_{0}^{T}\left[H_{0}(t)+V(t)\right] d t\right),
$$

where $X \equiv \mathcal{T} \exp \left(-i \int_{0}^{T} H_{0}(t)\right)$ satisfies $X^{N}=1$ for some integer $N$, and we assume that $H_{0}$ can be written as a sum $H_{0}(t)=\sum_{i} h_{i}(t)$ of terms acting on single sites $i$. Define $\lambda \equiv\|V\|_{1}$. Then, there exists a sequence of quasilocal $A_{n}$ such that, defining $\mathcal{U}_{n}=e^{-i A_{n}} \cdots e^{-i A_{1}}$, we have

$\mathcal{U}_{n} U_{\mathrm{f}} \mathcal{U}_{n}^{\dagger}=X \mathcal{T} \exp \left(-i \int_{0}^{1}\left[D_{n}+E_{n}+V_{n}(t)\right] d t\right)$,

where $\left[D_{n}, X\right]=0 ; D_{n}, E_{n}$ are independent of time; and

$$
\begin{gathered}
\left\|V_{n}\right\|_{n}, \quad\left\|E_{n}\right\|_{n} \leq 2 K_{n} \lambda^{n}, \\
\left\|A_{n}\right\|_{n} \leq(N+1) K_{n} \lambda^{n}, \\
\left\|D_{n}-D_{n-1}\right\|_{n} \leq K_{n} \lambda^{n},
\end{gathered}
$$

where we have defined $\lambda \equiv\|V\|_{1}$, and

$$
\begin{aligned}
K_{n} & =C^{n-1} \prod_{k=1}^{n-1} m(k), \\
C & =2(N+3)(N+4), \\
m(n) & =\frac{18}{\kappa_{n+1}\left(\kappa_{n}-\kappa_{n+1}\right)} .
\end{aligned}
$$

These bounds hold provided that $n \leq n_{*}$, with

$$
\begin{aligned}
& n_{*}=\frac{\lambda_{0} / \lambda}{\left[1+\log \left(\lambda_{0} / \lambda\right)\right]^{3}}, \\
& \lambda_{0}=(36 C)^{-1},
\end{aligned}
$$

and provided that

$$
\lambda<\frac{\mu}{N+3}, \quad \mu \approx 0.07 .
$$

Theorem 1 follows from Theorem $1^{\prime}$ because $n_{*}$ is chosen such that $n \leq n_{*}$ implies $\operatorname{Cm}(n) \leq(1 / 2 \lambda)$. It then follows that $K_{n+1} \lambda^{n+1} /\left(K_{n} \lambda^{n}\right)=\operatorname{Cm}(n) \lambda \leq \frac{1}{2}$ and hence that $K_{n} \lambda^{n} \leq \lambda / 2^{n-1}$. Moreover, we obtain Eq. (9) by 
summing Eq. (A10), from which we see $\left\|D_{n}-D_{1}\right\|_{n} \leq$ $\sum_{k=2}^{\infty} K_{k} \lambda^{k} \leq K_{2} \lambda^{2} \sum_{k=2}^{\infty}\left(\frac{1}{2}\right)^{k-2}=2 K_{2} \lambda^{2}=2 C m(1) \lambda^{2} \approx$ $2.9 \lambda^{2} / \lambda_{0}$. (Here, we use the fact that $\|\cdot\|_{n+1} \leq\|\cdot\|_{n}$.)

In the next subsections, we give a proof of Theorem $1^{\prime}$.

\section{Iterative construction}

The idea is to construct the $D_{n}, V_{n}, E_{n}, A_{n}$ discussed above iteratively. In other words, suppose that at the $n$th step, we have

$$
\mathcal{U}_{n} U_{\mathrm{f}} \mathcal{U}_{n}^{\dagger} \equiv U_{\mathrm{f}}^{(n)}=X \mathcal{T} \exp \left(-i \int_{0}^{1} \mathcal{H}_{n}(t) d t\right),
$$

where $\mathcal{H}_{n}(t)=F_{n}+V_{n}(t)$, with $F_{n}=\int_{0}^{T} \mathcal{H}_{n}(t) d t$ being time independent. We choose to separate the timeindependent piece $F_{n}$ according to $F_{n}=D_{n}+E_{n}$, where $D_{n}=\left\langle F_{n}\right\rangle$, and we have defined the symmetrization

$$
\langle O\rangle=\frac{1}{N} \sum_{k=0}^{N-1} X^{-k} O X^{k}
$$

In particular, this implies that $\left[D_{n}, X\right]=0$ and $\left\langle D_{n}\right\rangle=D_{n}$, and therefore $\left\langle E_{n}\right\rangle=\left\langle F_{n}\right\rangle-\left\langle D_{n}\right\rangle=D_{n}-D_{n}=0$.

We now introduce a local unitary $\mathcal{A}_{n}=e^{-i A_{n}}$, which we use to rotate the Floquet operator $U_{\mathrm{f}}^{(n)}$, giving a new Floquet operator

$U_{\mathrm{f}}^{(n+1)} \equiv \mathcal{A}_{n} U_{\mathrm{f}}^{(n)} \mathcal{A}_{n}^{\dagger}=X \mathcal{T} \exp \left(-i \int_{0}^{1} \mathcal{H}_{n+1}(t) d t\right)$.

The ultimate goal, decomposing $\mathcal{H}_{n+1}(t)=D_{n+1}+$ $E_{n+1}+V_{n+1}(t)$ as before, is to ensure that the residual error terms $E_{n+1}$ and $V_{n+1}$ are much smaller than $E_{n}$ and $V_{n}$. This goal is achieved in two separate steps. The first step ensures that $E_{n+1}$ is small [that is, the timeindependent part of $\mathcal{H}_{n+1}(t)$ nearly commutes with $\left.X\right]$, and the second step ensures that $V_{n+1}$ is small.

Step 1.-This step proceeds similarly to the recursion relation of Abanin et al. [74] for the time-independent case (Sec. 5.4 of Ref. [74]). There, the recursion relation was designed to make the Hamiltonian commute with its zeroth order version. This is analogous to our present goal of making the Floquet operator commute with $X$. Here, we adapt the analysis of Ref. [74] to the Floquet case.

We observe that

$$
\begin{aligned}
U_{\mathrm{f}}^{(n+1)} & =\mathcal{A}_{n} U_{\mathrm{f}}^{(n)} \mathcal{A}_{n}^{\dagger} \\
& =X\left[X^{\dagger} \mathcal{A}_{n} X \times \mathcal{T} \exp \left(-i \int_{0}^{1} \mathcal{H}_{n}(t) d t\right) \times \mathcal{A}_{n}^{\dagger}\right]
\end{aligned}
$$

$$
\begin{gathered}
=X\left[e^{-X^{\dagger} i A_{n} X} \times \mathcal{T} \exp \left(-i \int_{0}^{1} \mathcal{H}_{n}(t) d t\right) \times e^{i A_{n}}\right] \\
=X \times \mathcal{T} \exp \left(-i \int_{0}^{1} \mathcal{H}_{n}^{\prime}(t) d t\right),
\end{gathered}
$$

where

$$
\mathcal{H}_{n}^{\prime}(t)= \begin{cases}\frac{1}{a}\left(-A_{n}\right) & 0 \leq t \leq a \\ \frac{1}{1-2 a} \mathcal{H}_{n}\left(\frac{t-a}{1-2 a}\right) & a \leq t \leq(1-a) \\ \frac{1}{a}\left(X^{\dagger} A_{n} X\right) & (1-a) \leq t \leq 1\end{cases}
$$

(for some constant $a \in[0,1 / 2]$ which can be chosen arbitrarily). Let us decompose $\mathcal{H}_{n}^{\prime}(t)=D_{n}^{\prime}+V_{n}^{\prime}(t)$, where $D_{n}^{\prime}=\frac{1}{T} \int_{0}^{1} \mathcal{H}_{n}^{\prime}(t)$. Our goal is to ensure that the timeindependent part $D_{n}^{\prime}$ commutes with $X$. It turns out that this can actually be achieved exactly, and in particular, we can choose $A_{n}$ such that $D_{n}^{\prime}=D_{n}$.

To this end, we first observe that

$$
D_{n}^{\prime}=D_{n}+E_{n}+X^{\dagger} A_{n} X-A_{n} .
$$

We now claim that $D_{n}^{\prime}=D_{n}$ if we choose

$$
A_{n}:=\frac{1}{N} \sum_{k=0}^{N-1} \sum_{p=0}^{k} E_{n}^{(p)}, \quad E_{n}^{(p)}=X^{-p} E X^{p} .
$$

To see this, note that, by construction,

$$
\begin{aligned}
X^{\dagger} A_{n} X-A_{n} & =\frac{1}{N} \sum_{k=0}^{N-1} \sum_{p=0}^{k}\left[E_{n}^{(p+1)}-E_{n}^{(p)}\right] \\
& =\frac{1}{N} \sum_{k=0}^{N-1}\left[E_{n}^{(k+1)}-E_{n}\right] \\
& =-E_{n}+\left\langle E_{n}\right\rangle \\
& =-E_{n},
\end{aligned}
$$

since $\left\langle E_{n}\right\rangle=0$.

Step 2.-The next step is to find a new time-dependent Hamiltonian $\mathcal{H}_{n+1}(t)$ that gives the same unitary evolution as $\mathcal{H}_{n}^{\prime}(t)$ over the time interval $[0,1]$ while making the time-dependent part smaller. In other words, making the decomposition $\mathcal{H}_{n+1}(t)=D_{n+1}+E_{n+1}+V_{n+1}(t)$ as before, the goal is to make $V_{n+1}$ small. In fact, this is precisely the problem already considered by Abanin et al. [74], and we can use the procedure described in Sec. 4.1 of that paper.

One might worry whether step 2 undoes the good work done by step 1 . In other words, does making $V_{n+1}$ small 
come at the cost of making $E_{n+1}$ larger again? However, this turns out not to be a problem, as the bounds we derive below will make clear.

\section{Bounds on error terms}

Now, we derive bounds that quantify the success of the iterative procedure described in the previous subsection at making the residual error terms $E_{n}$ and $V_{n}$ small. The analysis proceeds in a way similar to that of Abanin et al. [74]. We define

$$
\begin{array}{ll}
d(n)=\left\|D_{n}\right\|_{n}, & v(n)=\left\|V_{n}\right\|_{n}, \quad v^{\prime}(n)=\left\|V_{n}^{\prime}\right\|_{n}, \\
e(n)=\left\|E_{n}\right\|_{n}, & \delta d(n)=\left\|D_{n+1}-D_{n}\right\|_{n+1} . \quad \text { (A28) }
\end{array}
$$

First of all, from Eq. (A23), we have a bound on $A_{n}$ :

$$
\left\|A_{n}\right\|_{n} \leq \frac{N+1}{2} e(n) .
$$

From Eq. (A21), we observe that

$$
V_{n}^{\prime}(t)= \begin{cases}\frac{1}{a}\left(-A_{n}\right)-D_{n} & 0 \leq t \leq a \\ \frac{1}{1-2 a}\left[2 a D_{n}+E_{n}+V_{n}\left(\frac{t-a}{1-2 a}\right)\right] & a \leq t \leq(1-a) \\ \frac{1}{a}\left(X^{\dagger} A_{n} X\right)-D_{n} & (1-a) \leq t \leq 1,\end{cases}
$$

and hence

$$
v^{\prime}(n) \leq 2\left\|A_{n}\right\|_{n}+\left\|E_{n}\right\|_{n}+\left\|V_{n}\right\|_{n}+4 a\left\|D_{n}\right\|_{n} .
$$

Thus, we can send $a \rightarrow 0$ to give [using Eq. (A29)]

$$
v^{\prime}(n) \leq(N+2) e(n)+v(n) .
$$

Then, as our construction of $\mathcal{H}_{n+1}$ from $\mathcal{H}_{n}^{\prime}$ is the one described in Sec. 4.1 of Abanin et al., we can use their bounds,

$$
\begin{array}{r}
\left\|D_{n+1}+E_{n+1}-D_{n}\right\|_{n+1} \leq \epsilon_{n} / 2, \\
v(n+1) \leq \epsilon_{n},
\end{array}
$$

where

$$
\begin{aligned}
\epsilon_{n} & =m(n) v^{\prime}(n)\left(d(n)+2 v^{\prime}(n)\right), \\
m(n) & =\frac{18}{\left(\kappa_{n+1}-\kappa_{n}\right) \kappa_{n+1}} .
\end{aligned}
$$

These bounds hold provided that

$$
3 v^{\prime}(n) \leq \kappa_{n}-\kappa_{n+1} .
$$

Since $D_{n+1}-D_{n}=\left\langle D_{n+1}+E_{n+1}-D_{n}\right\rangle$, we see that

$$
\delta d(n) \leq\left\|D_{n+1}+E_{n+1}-D_{n}\right\|_{n+1} \leq \epsilon_{n} / 2
$$

and

$$
\begin{aligned}
e(n+1) \leq & \left\|D_{n+1}+E_{n+1}-D_{n}\right\|_{n+1} \\
& +\left\|D_{n+1}-D_{n}\right\|_{n+1} \leq \epsilon_{n} .
\end{aligned}
$$

\section{Proof of Theorem $\mathbf{1}^{\prime}$ by induction}

The idea now is to apply the bounds of the previous subsection recursively to give bounds expressed in terms of the original Floquet operator,

$$
\begin{aligned}
U_{\mathrm{f}}=U_{\mathrm{f}}^{(1)} & =\mathcal{T} \exp \left(-i \int_{0}^{1}\left[H_{0}(t)+V(t)\right]\right) \\
& =X \mathcal{T} \exp \left(-i \int_{0}^{1} V_{\text {int }}(t) d t\right),
\end{aligned}
$$

and, in particular, the quantity $\lambda \equiv\left\|V_{\text {int }}\right\|_{1}=\|V\|_{1}$. First of all, we write $\mathcal{H}_{1}(t) \equiv V_{\text {int }}(t)=F_{1}+V_{1}(t)$, where $F_{1}=$ $\int_{0}^{1} V_{\text {int }}(t) d t$, and then separate $F_{1}=D_{1}+E_{1}$, where $D_{1}=\left\langle F_{1}\right\rangle$. We note that $\left\|F_{1}\right\|_{1} \leq \lambda$, which implies that $v(1) \leq\left\|V_{\text {int }}\right\|_{1}+\left\|F_{1}\right\|_{1} \leq 2 \lambda$, and $d(1) \leq \lambda$. In turn, this gives $e(1) \leq\left\|D_{1}\right\|_{1}+\left\|F_{1}\right\|_{1} \leq 2 \lambda$.

Now, we proceed by induction. Suppose that we have some $n$ such that, for all $1 \leq k \leq n$, we have

$$
e(k), v(k) \leq 2 K_{k} \lambda^{k},
$$

and for all $1 \leq k<n$,

$$
\delta d(k) \leq K_{k+1} \lambda^{k+1}
$$

where the coefficients $K_{k}$ satisfy $K_{k+1} / K_{k} \leq(1 / 2 \lambda)$. (The preceding discussion shows that this induction condition is satisfied for $n=1$ with $K_{1}=1$.)

Then, from Eq. (A32), we find that

$$
v^{\prime}(n) \leq 2 c_{N} K_{n} \lambda^{n}, \quad c_{N}=N+3,
$$

and hence

$$
\epsilon_{n} \leq m(n) 2 c_{N} K_{n} \lambda^{n}\left(d(n)+2 c_{N} K_{n} \lambda^{n}\right) .
$$

We note that the triangle inequality and the fact that $\|\cdot\|_{n}$ decreases with $n$ ensures that $d(n+1)-d(n) \leq \delta d(n)$. Hence, we can bound $d(n)$ by 


$$
\begin{aligned}
d(n) & \leq d(1)+\sum_{k=1}^{n-1} \delta d(k) \\
& \leq \lambda+\sum_{k=1}^{n-1} K_{k+1} \lambda^{k+1} \\
& =\sum_{k=1}^{n} K_{k} \lambda^{k} \\
& \leq \sum_{k=1}^{n} \lambda\left(\frac{1}{2}\right)^{k-1} \\
& \leq 2 \lambda .
\end{aligned}
$$

In Eq. (A49), we used the inequality $K_{k+1} / K_{k} \leq 1 /(2 \lambda)$. This same inequality also ensures that $K_{n} \lambda^{n} \leq \lambda$, so inserting this into Eq. (A45) gives

$$
\begin{aligned}
\epsilon_{n} & \leq m(n) 2 c_{N} K_{n}\left(2+2 c_{N}\right) \lambda^{n+1} \\
& \equiv 2 C m(n) K_{n} \lambda^{n+1} \\
& \equiv K_{n+1} \lambda^{n+1} .
\end{aligned}
$$

Here, we chose

$$
K_{n+1}=C m(n) K_{n}, \quad C=2 c_{N}\left(1+c_{N}\right) .
$$

Next, we need to examine the conditions under which Eq. (A37) holds. Given the bounds on $v^{\prime}(n)$ and using the inequality $K_{n} \lambda^{n} \leq \lambda(1 / 2)^{n-1}$, it is sufficient to demand that

$$
3 c_{N}(1 / 2)^{n-1} \lambda \leq \kappa_{n+1}-\kappa_{n},
$$

or, in other words,

$\lambda \leq \frac{1}{3 c_{N}} \max _{n \in \mathbb{N}}\left[2^{n-1}\left(\kappa_{n+1}-\kappa_{n}\right)\right]=\frac{1}{3 c_{N}}\left(\kappa_{2}-\kappa_{1}\right) \approx \frac{0.14 \kappa_{1}}{N+3}$.

Provided that Eq. (A54) holds, we then find that

$$
\delta d(n), v(n+1) / 2, e(n+1) / 2 \leq K_{n+1} \lambda^{n+1} .
$$

Therefore, we can continue the induction provided that $K_{n+1} / K_{n} \leq \frac{1}{2 \lambda}$. Since $K_{n+1} / K_{n}=C m(n)$, this is true provided that $n \leq n_{*}$. This completes the proof of Theorem 1'.

\section{APPENDIX B: PROOF OF PHASE WIDENING WHEN A U(1) SYMMETRY IS SPONTANEOUSLY BROKEN}

Here, we intend to prove the claim made in Sec. IV A above that the expectation value

$$
\operatorname{Tr}\left(\rho_{X} e^{i t K} \Phi e^{-i t K}\right) \equiv g_{X}(t)
$$

must be independent of time $t$, where we have defined $K \equiv D-\mu L$ and $\rho_{X} \equiv \lim _{\epsilon \rightarrow 0^{+}}(1 / \mathcal{Z}) e^{-\beta(K+\epsilon X)}$. The idea is to make a connection with the results of Ref. [42]; however, these results were expressed in terms of two-point correlation functions and did not have the $\epsilon X$ term in the definition of the density matrix. To make a connection, we assume that the symmetric density matrix $\rho=(1 / \mathcal{Z}) e^{-\beta K}$ can be recovered by symmetrizing a symmetry-breaking state,

$$
\rho=\frac{1}{2 \pi} \int_{0}^{2 \pi} e^{-i \theta L} \rho_{X} e^{i \theta L} d \theta
$$

and that the symmetry-breaking state $\rho_{X}$ is short-range correlated. Now, we calculate the two-point correlation function [where $\Phi(x)$ and $\Phi(y)$ are two operators acting at different spatial locations $x$ and $y$ ]

$$
\begin{aligned}
f(t)= & \operatorname{Tr}\left[\rho e^{i t K} \Phi(x) e^{i t K} \Phi^{\dagger}(y)\right] \\
= & \frac{1}{2 \pi} \int_{0}^{2 \pi} d \theta \operatorname{Tr}\left[e^{-i \theta L} \rho_{X} e^{i \theta L} e^{i t K} \Phi(x) e^{-i t K} \Phi(y)\right] \\
= & \frac{1}{2 \pi} \int_{0}^{2 \pi} d \theta \operatorname{Tr}\left[\rho_{X} e^{i t K}\left\{e^{i \theta L} \Phi(x) e^{-i \theta L}\right\}\right. \\
& \left.\times e^{-i t K}\left\{e^{i \theta L} \Phi^{\dagger}(y) e^{-i \theta L}\right\}\right] \\
= & \left.\operatorname{Tr}\left[\rho_{X}\left\{e^{-i t K} \Phi(x) e^{i t K}\right\} \Phi^{\dagger}(y)\right\}\right] \\
= & g_{X}(t)\left[g_{X}(0)\right]^{*},
\end{aligned}
$$

where we used the fact that $L$ and $K$ commute and that $e^{i \theta L} \Phi e^{-i \theta L}=e^{i \theta} \Phi$. In the last line, we sent $|x-y| \rightarrow \infty$ and used the assumption that $\rho_{X}$ has short-range correlations.

Now, the theorem of Ref. [42] rigorously proves that the function $f(t)$ must be independent of time. Hence, unless $g_{X}(0)=0$, we conclude that $g_{X}(t)$ must be independent of time. [If $g_{X}(0)=0$ but $g_{X}(t)$ is not independent of time, then there must be some $t$ such that $g_{X}(t) \neq 0$. Then, we can just relabel the time coordinate so that $g_{X}(0) \neq 0$ and repeat the argument.]

\section{APPENDIX C: OPEN SYSTEMS}

In this section, we elaborate on our hypothesis for open systems introduced in Sec. VI above, namely, that in a large 
class of systems, the steady state will have low energy. First, we need to clarify what we mean by "energy" and "steady state" in the Floquet context. Let $H_{S}(t)$ be the time evolution of the system alone (not taking into account the coupling to the environment). We define the Floquet operator $U_{\mathrm{f}}=\mathcal{T} \exp \left(-i \int_{0}^{T} H_{S}(t) d t\right)$. Recall that in the regime discussed in Sec. III, where $\lambda$ as defined there satisfies $\lambda T \ll 1$, we can write $H_{S}(t)=\tilde{H}_{S}(t)+V(t)$. Here, $V(t)$ is a very weak residual perturbation, and $\tilde{H}_{S}(t)$ is such that, if we define the approximate Floquet operator by $\widetilde{U_{\mathrm{f}}}=\mathcal{T} \exp \left(-i \int_{0}^{T} \tilde{H}_{S}(t)\right)$, then it can be expressed, following a local unitary time-independent change of basis (which we will set to 1 here for notational simplicity), as $\widetilde{U_{\mathrm{f}}}=X e^{-i D T}$, where $X^{2}=1$ and $D$ is a quasilocal Hamiltonian $D$ that commutes with $X$. In particular, we have $\widetilde{U}_{\mathrm{f}}^{2}=e^{-2 i D T}$. This implies that we can make a time-dependent local unitary change of basis $W(t)$, periodic with period $2 T$ and satisfying $W(0)=1$, such that the transformed Hamiltonian, which is related to $\tilde{H}_{S}(t)$ according to

$$
\tilde{H}_{S}^{\prime}=W H_{S} W^{\dagger}+i\left[\partial_{t} W\right] W^{\dagger},
$$

is time independent and equal to $D$. Therefore, in this new reference frame, it is clear that we should refer to the expectation value of $D$ as "energy." We emphasize that we have not eliminated the time dependence completely: Even in the new reference frame, the residual driving term $V(t)$, as well as any couplings to the environment, will still be time dependent. (Because of the time-dependent change of basis, the latter will gain a time dependence even if it was originally time independent.)

The steady state is now determined by some balance between the residual periodic driving $V(t)$, the classical noise, and the coupling to the environment. We leave a detailed analysis of this open system process for future work, but we expect that, in a suitable regime, the energy density of the steady state will be low. (For one study of steady states of many-body Floquet systems coupled to a bath, see Ref. [98].) We now explain why this implies oscillations (which are observed in the original reference frame, not the rotating one defined above).

Consider a short-range correlated steady state $\rho$ whose energy density with respect to $D$ is small. Recall that in Sec. III A, we argued that if $\rho$ is a thermal state, it must spontaneously break the symmetry generated by $X$, and it follows that under $\widetilde{U_{\mathrm{f}}}$, it oscillates at twice the drive frequency. Of course, for an open system, the steady state need not be thermal, and time evolution of the open system is not exactly given by $\widetilde{U_{\mathrm{f}}}$. However, as we prove in Appendix D, even nonthermal states must fail to be invariant under the symmetry $X$ if their energy density with respect to $D$ is sufficiently small, provided that they satisfy a physically reasonable "thermalizability" condition. Moreover, if $\lambda T \ll 1$ (so that we can approximate $\widetilde{U_{\mathrm{f}}} \approx X$ ), and the coupling to the environment is sufficiently weak, then the resulting state after one time period is approximately given by $X \rho X^{\dagger}$, which according to the preceding discussion is not the same as $\rho$. (We make this argument more precise in Appendix D.) Thus, provided that the energy of the steady state is sufficiently small, it does not return to itself after one time period, and oscillations with period $2 T$ will be observed.

Generic baths will destroy continuous-time time crystals. The difference from the discrete-time case is the existence of an extra variable characterizing thermal states of $D$, namely, the chemical potential $\mu$. This extra variable is needed because of the presence of the hidden $\mathrm{U}(1)$ symmetry in the continuous-time regime. (There is no analogous variable when the hidden symmetry is discrete.) Thus, one certainly cannot make any statement that all lowenergy states of $D$ oscillate because, in particular, a thermal state of $D$ in which the electrochemical potential $\mu-u=0$ does not oscillate. A coupling to a generic bath will not preserve the hidden U(1) symmetry, and thus, to the extent that the steady state of an open system process is close to a thermal state of $D$, we in fact expect it to have $\mu-u=0$ since this corresponds to minimizing the free energy.

In principle, one could fine-tune the bath so that it respects the symmetry. This would allow the time crystal to survive, but it is clearly contrived. One might wonder whether the bath itself could also prethermalize: If we could consider the bath to be included in the Hamiltonian (34), then it could have an approximate U(1) symmetry along with the rest of the system. This would require the local terms in the bath Hamiltonian to be much smaller than the coupling $u$ in Eq. (34). However, for most of the physically relevant baths that one would want to consider (for example, phonons), the local terms in the bath Hamiltonian are in fact unbounded.

\section{APPENDIX D: SPONTANEOUS SYMMETRY BREAKING FOR NONTHERMAL STATES}

Let $D$ be a quasilocal Hamiltonian for which the thermal states spontaneously break an on-site $\mathbb{Z}_{N}$ symmetry generated by $X$ for energy densities $e<e_{c}$. More precisely, what we mean is the following, where we define the local distance between two states on a region $A$ according to

$$
\left\|\rho_{1}-\rho_{2}\right\|_{A}=\left\|\left(\rho_{1}\right)_{A}-\left(\rho_{2}\right)_{A}\right\|_{1},
$$

where $\|\cdot\|_{1}$ is the trace norm, and $(\rho)_{A}=\operatorname{Tr}_{A^{c}} \rho$ is the reduced state of $\rho$ on $A$.

Assumption 1 (Spontaneous symmetry breaking). There exists some finite region $A$ and some $\gamma>0$ such that, for any short-range correlated thermal state $\rho_{\tau}$ with energy density $e<e_{c}$, we have $\left\|\rho_{\tau}-X^{k} \rho_{\tau} X^{-k}\right\|_{A} \geq \gamma$ for all $0<k<N$. 
Now, let $\rho$ be any state (not necessarily thermal) such that the energy density $\epsilon \equiv\langle D\rangle_{\rho} / V<\epsilon_{c}$ (with $V$ the volume of the system.) We assume the following thermalizability condition, which roughly states that $\rho$ can thermalize when time evolved under $D$.

Assumption 2 (Thermalizability). There exist a time $t_{1}$ and a short-range correlated thermal state $\rho_{\tau}$ with the same energy density as $\rho$, such that $\left\|\rho\left(t_{1}\right)-\rho_{\tau}\right\|_{A} \leq \gamma / 8$, where $\rho(t)=e^{-i D t_{1}} \rho e^{i D t_{1}}$.

From Assumptions 1 and 2, we derive the following lemma, which quantifies the sense in which the state $\rho$ must break the symmetry.

Lemma 1. There exists a finite region $A^{\prime}$ such that $\left\|\rho-X^{k} \rho X^{-k}\right\|_{A^{\prime}} \geq 3 \gamma / 4$.

Proof. From the triangle inequality, it follows that

$$
\begin{aligned}
\| \rho\left(t_{1}\right) & -X^{k} \rho\left(t_{1}\right) X^{-k} \|_{A} \\
\geq & \left\|\rho_{\tau}-X^{k} \rho_{\tau} X^{-k}\right\|_{A}-\| \rho\left(t_{1}\right)-X^{k} \rho\left(t_{1}\right) X^{-k} \\
& -\left(\rho_{\tau}-X^{k} \rho_{\tau} X^{-k}\right) \|_{A} \\
\geq & \gamma-2 \gamma / 8 \\
= & 3 \gamma / 4 .
\end{aligned}
$$

Using the characterization of the trace norm as

$$
\|\rho\|_{1}=\sup _{\hat{o}:\|\hat{o}\|=1}\left|\langle\hat{o}\rangle_{\rho}\right|,
$$

it follows that there exists an operator $\hat{o}_{A}$ supported on $A$, with $\left\|\hat{o}_{A}\right\|=1$, such that $\left|\left\langle X^{-k} \hat{o}_{A} X^{k}-\hat{o}_{A}\right\rangle_{\rho\left(t_{1}\right)}\right| \geq 3 \gamma / 4$. Now, since $D$ is quasilocal, it must obey a Lieb-Robinson bound $[99,100]$, which implies that there exists a local operator $\hat{O}_{A^{\prime}}$ supported on a finite region $A^{\prime}$ such that $\left\|\hat{o}\left(t_{1}\right)-\hat{O}_{A^{\prime}}\right\| \leq \gamma / 8$, where $\hat{o}\left(t_{1}\right)=e^{i D t_{1}} \hat{o} e^{-i D t_{1}}$. Hence, we see that

$$
\begin{aligned}
& \left|\left\langle X^{-k} \hat{O}_{A^{\prime}} X^{k}-\hat{O}_{A^{\prime}}\right\rangle_{\rho}\right| \\
& \geq-\gamma / 4+\left|\left\langle X^{-k} \hat{o}_{A}\left(t_{1}\right) X^{k}-\hat{o}_{A}\left(t_{1}\right)\right\rangle_{\rho}\right| \\
& =-\gamma / 4+\left|\left\langle X^{-k} \hat{o}_{A} X^{k}-\hat{o}_{A}\right\rangle_{\rho\left(t_{1}\right)}\right| \\
& \geq-\gamma / 4+3 \gamma / 4 \\
& =\gamma / 2 .
\end{aligned}
$$

To get to Eq. (D9), we used the fact that $X$ and $D$ commute. The lemma follows.

Now, consider a system which, in isolation, would evolve under a time-dependent Hamiltonian $H(t)$, which is periodic with period $T$. We assume that $H(t)$ exhibits the prethermalization phenomena discussed in the main text. In other words, we assume that the Floquet operator can be approximated according to $U_{\mathrm{f}} \approx \widetilde{U_{\mathrm{f}}}=X e^{-i D T}$, where $D$ is quasilocal and commutes with $X$, and where $U_{\mathrm{f}}$ is close to $\widetilde{U_{\mathrm{f}}}$ in the sense that

$$
\left\|U_{\mathrm{f}}^{\dagger} O_{A^{\prime}} U_{\mathrm{f}}-\widetilde{U}_{\mathrm{f}}^{\dagger} O_{A^{\prime}} \widetilde{U}_{\mathrm{f}}\right\| \leq \frac{\gamma}{8}\left\|O_{A^{\prime}}\right\|
$$

for any operator $O_{A^{\prime}}$ supported on $A^{\prime}$.

Let $\rho_{\text {open }}(t)$ be the reduced state of the system (tracing out the bath) at time $t$, taking into account the systembath coupling, and we assume that $\rho_{\text {open }}(0) \equiv \rho$ satisfies Assumption 2 above. We assume the coupling to the bath is sufficiently weak, in the following sense:

Assumption 3 (Weak coupling). For any time $0 \leq t \leq T$, we have $\left\|\rho_{\text {open }}^{\text {int }}(t)-\rho\right\|_{A^{\prime}} \leq \gamma / 8$.

Here, we defined the interaction picture state $\rho_{\text {open }}^{\text {int }}(t)=U(0, t)^{-1} \rho_{\text {open }}(t) U(0, t)$, where $U(0, t)$ is the time evolution generated by $H(t)$. If we were to set the coupling to the bath to zero, then the state $\rho_{\text {open }}^{\text {int }}(t)$ would be constant in time, so Assumption 3 corresponds to weak coupling. Finally, we assume that the strength of $D T$ is small enough so that the following assumption holds.

Assumption 4. For any observable $O_{A^{\prime}}$ supported on $A^{\prime}$, we have

$$
\left\|e^{-i D T} O_{A^{\prime}} e^{i D T}-O_{A^{\prime}}\right\| \leq \frac{\gamma}{8}\left\|O_{A^{\prime}}\right\| .
$$

This assumption will always be true in the regime of interest, $\lambda T \ll 1$ (where $\lambda$ is as defined in Sec. II) because $\|D\|_{n_{*}}$ is $O(\lambda)$ [see Eq. (9) in Theorem 1].

From the above assumptions, we can now derive our main result.

Theorem 3.

$$
\left\|\rho_{\text {open }}(T)-\rho\right\|_{A^{\prime}} \geq \gamma / 8 \text {. }
$$

Proof.

$$
\begin{aligned}
& \left\|\rho_{\text {open }}(T)-\rho\right\|_{A^{\prime}} \\
& \quad=\left\|U_{\mathrm{f}} \rho_{\mathrm{open}}^{\mathrm{int}}(T) U_{\mathrm{f}}^{\dagger}-\rho\right\|_{A^{\prime}} \\
& \geq-\gamma / 8+\left\|\tilde{U}_{\mathrm{f}} \rho_{\mathrm{open}}^{\mathrm{int}}(T) \tilde{U}_{\mathrm{f}}^{\dagger}-\rho\right\|_{A^{\prime}} \\
& \quad=-\gamma / 8+\left\|e^{-i D T} \rho_{\mathrm{open}}^{\mathrm{int}}(T) e^{i D T}-X^{\dagger} \rho X\right\|_{A^{\prime}} \\
& \geq-\gamma / 8-\gamma / 8+\left\|\rho_{\mathrm{open}}^{\mathrm{int}}(T)-X^{\dagger} \rho X\right\|_{A^{\prime}} \\
& \geq-\gamma / 8-\gamma / 8-\gamma / 8+\left\|\rho-X^{\dagger} \rho X\right\|_{A^{\prime}}
\end{aligned}
$$




$$
\begin{aligned}
& \geq-\gamma / 8-\gamma / 8-\gamma / 8+\gamma / 2 \\
& =\gamma / 8
\end{aligned}
$$

In other words, the state of the open system at time $t=T$ and that at time $t=0$ are locally distinguishable. Particularly, for the stated assumptions, the state of the system does not synchronize with the drive, and timetranslation symmetry is spontaneously broken.

[1] Z.-C. Gu and X.-G. Wen, Tensor-Entanglement-Filtering Renormalization Approach and Symmetry-Protected Topological Order, Phys. Rev. B 80, 155131 (2009).

[2] F. Pollmann, A. M. Turner, E. Berg, and M. Oshikawa, Entanglement Spectrum of a Topological Phase in One Dimension, Phys. Rev. B 81, 064439 (2010).

[3] F. Pollmann, E. Berg, A. M. Turner, and M. Oshikawa, Symmetry Protection of Topological Phases in OneDimensional Quantum Spin Systems, Phys. Rev. B 85, 075125 (2012).

[4] L. Fidkowski and A. Kitaev, Effects of Interactions on the Topological Classification of Free Fermion Systems, Phys. Rev. B 81, 134509 (2010).

[5] X. Chen, Z.-C. Gu, and X.-G. Wen, Local Unitary Transformation, Long-Range Quantum Entanglement, Wave Function Renormalization, and Topological Order, Phys. Rev. B 82, 155138 (2010).

[6] X. Chen, Z.-C. Gu, and X.-G. Wen, Classification of Gapped Symmetric Phases in One-Dimensional Spin Systems, Phys. Rev. B 83, 035107 (2011).

[7] N. Schuch, D. Pérez-García, and I. Cirac, Classifying Quantum Phases Using Matrix Product States and Projected Entangled Pair States, Phys. Rev. B 84, 165139 (2011).

[8] L. Fidkowski and A. Kitaev, Topological Phases of Fermions in One Dimension, Phys. Rev. B 83, 075103 (2011).

[9] X. Chen, Z.-X. Liu, and X.-G. Wen, Two-Dimensional Symmetry-Protected Topological Orders and Their Protected Gapless Edge Excitations, Phys. Rev. B 84, 235141 (2011).

[10] X. Chen, Z.-C. Gu, Z.-X. Liu, and X.-G. Wen, Symmetry Protected Topological Orders and the Group Cohomology of Their Symmetry Group, Phys. Rev. B 87, 155114 (2013).

[11] M. Levin and Z.-C. Gu, Braiding Statistics Approach to Symmetry-Protected Topological Phases, Phys. Rev. B 86, 115109 (2012).

[12] A. Vishwanath and T. Senthil, Physics of ThreeDimensional Bosonic Topological Insulators: SurfaceDeconfined Criticality and Quantized Magnetoelectric Effect, Phys. Rev. X 3, 011016 (2013).

[13] C. Wang, A. C. Potter, and T. Senthil, Classification of Interacting Electronic Topological Insulators in Three Dimensions, Science 343, 629 (2014).
[14] A. Kapustin, Symmetry Protected Topological Phases, Anomalies, and Cobordisms: Beyond Group Cohomology, arXiv:1403.1467.

[15] Z.-C. Gu and X.-G. Wen, Symmetry-Protected Topological Orders for Interacting Fermions: Fermionic Topological Nonlinear $\sigma$ Models and a Special Group Supercohomology Theory, Phys. Rev. B 90, 115141 (2014).

[16] D. V. Else and C. Nayak, Classifying Symmetry-Protected Topological Phases through the Anomalous Action of the Symmetry on the Edge, Phys. Rev. B 90, 235137 (2014).

[17] F. J. Burnell, X. Chen, L. Fidkowski, and A. Vishwanath, Exactly Soluble Model of a Three-Dimensional SymmetryProtected Topological Phase of Bosons with Surface Topological Order, Phys. Rev. B 90, 245122 (2014).

[18] J. C. Wang, Z.-C. Gu, and X.-G. Wen, Field-Theory Representation of Gauge-Gravity Symmetry-Protected Topological Invariants, Group Cohomology, and Beyond, Phys. Rev. Lett. 114, 031601 (2015).

[19] M. Cheng, Z. Bi, Y.-Z. You, and Z.-C. Gu, Towards a Complete Classification of Symmetry-Protected Phases for Interacting Fermions in Two Dimensions, arXiv:1501.01313.

[20] M.Z. Hasan and C. L. Kane, Colloquium: Topological Insulators, Rev. Mod. Phys. 82, 3045 (2010).

[21] Xiao-Liang Qi and Shou-Cheng Zhang, Topological Insulators and Superconductors, Rev. Mod. Phys. 83, 1057 (2011).

[22] J. Maciejko, X.-L. Qi, A. Karch, and S.-C. Zhang, Fractional Topological Insulators in Three Dimensions, Phys. Rev. Lett. 105, 246809 (2010).

[23] A. M. Essin and M. Hermele, Classifying Fractionalization: Symmetry Classification of Gapped $\mathbb{Z}_{2}$ Spin Liquids in Two Dimensions, Phys. Rev. B 87, 104406 (2013).

[24] Y.-M. Lu and A. Vishwanath, Classification and Properties of Symmetry Enriched Topological Phases: A ChernSimons Approach with Applications to Z2 Spin Liquids, Phys. Rev. B 93, 155121 (2016).

[25] A. Mesaros and Y. Ran, Classification of Symmetry Enriched Topological Phases with Exactly Solvable Models, Phys. Rev. B 87, 155115 (2013).

[26] L.-Y. Hung and X.-G. Wen, Quantized Topological Terms in Weak-Coupling Gauge Theories with a Global Symmetry and Their Connection to Symmetry-Enriched Topological Phases, Phys. Rev. B 87, 165107 (2013).

[27] M. Barkeshli, P. Bonderson, M. Cheng, and Z. Wang, Symmetry, Defects, and Gauging of Topological Phases, arXiv:1410.4540 [Phys. Rev. X (to be published)].

[28] M. Cheng, M. Zaletel, M. Barkeshli, A. Vishwanath, and P. Bonderson, Translational Symmetry and Microscopic Constraints on Symmetry-Enriched Topological Phases: A View from the Surface, Phys. Rev. X 6, 041068 (2016).

[29] V. Khemani, A. Lazarides, R. Moessner, and S. L. Sondhi, Phase Structure of Driven Quantum Systems, Phys. Rev. Lett. 116, 250401 (2016).

[30] C. W. von Keyserlingk and S. L. Sondhi, Phase Structure of One-Dimensional Interacting Floquet Systems. I. Abelian Symmetry-Protected Topological Phases, Phys. Rev. B 93, 245145 (2016).

[31] D. V. Else and C. Nayak, Classification of Topological Phases in Periodically Driven Interacting Systems, Phys. Rev. B 93, 201103 (2016). 
[32] A. C. Potter, T. Morimoto, and A. Vishwanath, Topological Classification of Interacting $1 D$ Floquet Phases, Phys. Rev. X 6, 041001 (2016).

[33] R. Roy and F. Harper, Abelian Floquet SymmetryProtected Topological Phases in One Dimension, Phys. Rev. B 94, 125105 (2016).

[34] F. Wilczek, Quantum Time Crystals, Phys. Rev. Lett. 109, 160401 (2012).

[35] A. Shapere and F. Wilczek, Classical Time Crystals, Phys. Rev. Lett. 109, 160402 (2012).

[36] T. Li, Z.-X. Gong, Z.-Q. Yin, H. T. Quan, X. Yin, P. Zhang, L.-M. Duan, and X. Zhang, Space-Time Crystals of Trapped Ions, Phys. Rev. Lett. 109, 163001 (2012).

[37] P. Bruno Comment on "Quantum Time Crystals", Phys. Rev. Lett. 110, 118901 (2013).

[38] P. Bruno, Comment on Space-Time Crystals of Trapped Ions”, Phys. Rev. Lett. 111, 029301 (2013).

[39] P. Bruno, Impossibility of Spontaneously Rotating Time Crystals: A No-Go Theorem, Phys. Rev. Lett. 111, 070402 (2013).

[40] P. Noziéres, Time Crystals: Can Diamagnetic Currents Drive a Charge Density Wave into Rotation?, Eur. Phys. Lett. 103, 57008 (2013).

[41] G. E. Volovik, On the Broken Time Translation Symmetry in Macroscopic Systems: Precessing States and OffDiagonal Long-Range Order, JETP Lett. 98, 491 (2013).

[42] H. Watanabe and M. Oshikawa, Absence of Quantum Time Crystals, Phys. Rev. Lett. 114, 251603 (2015).

[43] D. V. Else, B. Bauer, and C. Nayak, Floquet Time Crystals, Phys. Rev. Lett. 117, 090402 (2016).

[44] C. W. von Keyserlingk and S. L. Sondhi, Phase Structure of One-Dimensional Interacting Floquet Systems. II. Symmetry-Broken Phases, Phys. Rev. B 93, 245146 (2016).

[45] C. W. von Keyserlingk, V. Khemani, and S. L. Sondhi, Absolute Stability and Spatiotemporal Long-Range Order in Floquet Systems, Phys. Rev. B 94, 085112 (2016).

[46] L. D'Alessio and M. Rigol, Long-Time Behavior of Isolated Periodically Driven Interacting Lattice Systems, Phys. Rev. X 4, 041048 (2014).

[47] A. Lazarides, A. Das, and R. Moessner, Equilibrium States of Generic Quantum Systems Subject to Periodic Driving, Phys. Rev. E 90, 012110 (2014).

[48] P. Ponte, A. Chandran, Z. Papić, and D. A. Abanin, Periodically Driven Ergodic and Many-Body Localized Quantum Systems, Ann. Phys. (Amsterdam) 353, 196 (2015).

[49] D. A. Abanin, W. De Roeck, and F. Huveneers, A Theory of Many-Body Localization in Periodically Driven Systems, Ann. Phys. (Amsterdam) 372, 1 (2016).

[50] P. Ponte, A. Chandran, Z. Papić, and D. A. Abanin, Periodically Driven Ergodic and Many-Body Localized Quantum Systems, Ann. Phys. (Amsterdam) 353, 196 (2015).

[51] P. Ponte, Z. Papić, F. Huveneers, and D. A. Abanin, Many-Body Localization in Periodically Driven Systems, Phys. Rev. Lett. 114, 140401 (2015).

[52] A. Lazarides, A. Das, and R. Moessner, Fate of Many-Body Localization Under Periodic Driving, Phys. Rev. Lett. 115, 030402 (2015).
[53] T. Iadecola, L. H. Santos, and C. Chamon, Stroboscopic Symmetry-Protected Topological Phases, Phys. Rev. B 92, 125107 (2015).

[54] D. M. Basko, I. L. Aleiner, and B. L. Altshuler, Metal Insulator Transition in a Weakly Interacting ManyElectron System with Localized Single-Particle States, Ann. Phys. (Amsterdam) 321, 1126 (2006).

[55] D. M. Basko, I. L. Aleiner, and B. L. Altshuler, On the Problem of Many-Body Localization, arXiv:cond-mat/ 0602510.

[56] V. Oganesyan and D. A. Huse, Localization of Interacting Fermions at High Temperature, Phys. Rev. B 75, 155111 (2007).

[57] M. Žnidarič, T. Prosen, and P. Prelovšek, Many-Body Localization in the Heisenberg XXZ Magnet in a Random Field, Phys. Rev. B 77, 064426 (2008).

[58] A. Pal and D. A. Huse, Many-Body Localization Phase Transition, Phys. Rev. B 82, 174411 (2010).

[59] J. H. Bardarson, F. Pollmann, and J. E. Moore, Unbounded Growth of Entanglement in Models of Many-Body Localization, Phys. Rev. Lett. 109, 017202 (2012).

[60] B. Bauer and C. Nayak, Area Laws in a Many-Body Localized State and Its Implications for Topological Order, J. Stat. Mech. (2013) P09005.

[61] M. Serbyn, Z. Papić, and D. A. Abanin, Universal Slow Growth of Entanglement in Interacting Strongly Disordered Systems, Phys. Rev. Lett. 110, 260601 (2013).

[62] M. Serbyn, Z. Papić, and D. A. Abanin, Local Conservation Laws and the Structure of the Many-Body Localized States, Phys. Rev. Lett. 111, 127201 (2013).

[63] D. A. Huse, R. Nandkishore, and V. Oganesyan, Phenomenology of Fully Many-Body-Localized Systems, Phys. Rev. B 90, 174202 (2014).

[64] E. Levi, M. Heyl, I. Lesanovsky, and J. P. Garrahan, Robustness of Many-Body Localization in the Presence of Dissipation, Phys. Rev. Lett. 116, 237203 (2016).

[65] M. H. Fischer, M. Maksymenko, and E. Altman, Dynamics of a Many-Body-Localized System Coupled to a Bath, Phys. Rev. Lett. 116, 160401 (2016).

[66] R. Nandkishore, S. Gopalakrishnan, and D. A. Huse, Spectral Features of a Many-Body-Localized System Weakly Coupled to a Bath, Phys. Rev. B 90, 064203 (2014).

[67] S. Gopalakrishnan and R. Nandkishore, Mean-Field Theory of Nearly Many-Body Localized Metals, Phys. Rev. B 90, 224203 (2014).

[68] S. Johri, R. Nandkishore, and R. N. Bhatt, Many-Body Localization in Imperfectly Isolated Quantum Systems, Phys. Rev. Lett. 114, 117401 (2015).

[69] R. Nandkishore, Many-Body Localization Proximity Effect, Phys. Rev. B 92, 245141 (2015).

[70] X. Li, S. Ganeshan, J. H. Pixley, and S. Das Sarma, Many Body Localization and Quantum Non-ergodicity in a Model with a Single-Particle Mobility Edge, Phys. Rev. Lett. 115, 186601 (2015).

[71] R. Nandkishore and S. Gopalakrishnan, Many Body Localized Systems Weakly Coupled to Baths, Ann. Phys. (Amsterdam), DOI: 10.1002/andp.201600181 (2016).

[72] K. Hyatt, J. R. Garrison, A. C. Potter, and B. Bauer, Many-Body Localization in the Presence of a Small Bath, Phys. Rev. B 95, 035132 (2017). 
[73] D. A. Abanin, W. De Roeck, and F. Huveneers, Exponentially Slow Heating in Periodically Driven Many-Body Systems, Phys. Rev. Lett. 115, 256803 (2015).

[74] D. A. Abanin, W. De Roeck, and W. W. Ho, Effective Hamiltonians, Prethermalization and Slow Energy Absorption in Periodically Driven Many-Body Systems, Phys. Rev. B 95, 014112 (2017).

[75] D. Abanin, W. De Roeck, F. Huveneers, and W. W. Ho, A Rigorous Theory of Many-Body Prethermalization for Periodically Driven and Closed Quantum Systems, arXiv:1509.05386.

[76] T. Kuwahara, T. Mori, and K. Saito, Floquet-Magnus Theory and Generic Transient Dynamics in Periodically Driven Many-Body Quantum Systems, Ann. Phys. (Amsterdam) 367, 96 (2016).

[77] T. Mori, T. Kuwahara, and K. Saito, Rigorous Bound on Energy Absorption and Generic Relaxation in Periodically Driven Quantum Systems, Phys. Rev. Lett. 116, 120401 (2016).

[78] M. Bukov, S. Gopalakrishnan, M. Knap, and E. Demler, Prethermal Floquet Steady States and Instabilities in the Periodically Driven, Weakly Interacting Bose-Hubbard Model, Phys. Rev. Lett. 115, 205301 (2015).

[79] E. Canovi, M. Kollar, and M. Eckstein, Stroboscopic Prethermalization in Weakly Interacting Periodically Driven Systems, Phys. Rev. E 93, 012130 (2016).

[80] M. Bukov, M. Heyl, D. A. Huse, and A. Polkovnikov, Heating and Many-Body Resonances in a Periodically Driven Two-Band System, Phys. Rev. B 93, 155132 (2016).

[81] J. Schwinger, Brownian Motion of a Quantum Oscillator, J. Math. Phys. 2, 407 (1961).

[82] L. V. Keldysh, Diagram Technique for Non-equilibrium Processes, Zh. Eksp. Teor. Fiz. 47, 1515 (1964) [Sov. Phys. JETP 20, 1018 (1965)].

[83] A. Kamenev, Many-Body Theory of Non-equilibrium Systems, arXiv:cond-mat/0412296.

[84] D. V. Else, B. Bauer, and C. Nayak (unpublished).

[85] D. Pekker, G. Refael, E. Altman, E. Demler, and V. Oganesyan, Hilbert-Glass Transition: New Universality of Temperature-Tuned Many-Body Dynamical Quantum Criticality, Phys. Rev. X 4, 011052 (2014).

[86] D. A. Huse, R. Nandkishore, V. Oganesyan, A. Pal, and S. L. Sondhi, Localization-Protected Quantum Order, Phys. Rev. B 88, 014206 (2013).
[87] R. Haag, Local Quantum Physics: Fields, Particles, Algebras (Springer, New York, 1996).

[88] F. Wilczek, Superfluidity and Space-Time Translation Symmetry Breaking, Phys. Rev. Lett. 111, 250402 (2013).

[89] C. J. Pethick and H. Smith, Bose-Einstein Condensation in Dilute Gases (Cambridge University Press, Cambridge, 2008).

[90] A. Nicolis and F. Piazza, Spontaneous Symmetry Probing, J. High Energy Phys. 06 (2012) 025.

[91] E. Castillo, B. Koch, and G. Palma, On the Dynamics of Fluctuations in Time Crystals, arXiv:1410.2261 [Phys. Rev. Lett. (to be published)].

[92] M. Thies, Semiclassical Time Crystal in the Chiral GrossNeveu Model, arXiv:1411.4236 [Phys. Rev. D (to be published)].

[93] P. Bruno, Impossibility of Spontaneously Rotating Time Crystals: A No-Go Theorem, Phys. Rev. Lett. 111, 070402 (2013).

[94] M. Schreiber, S. S. Hodgman, P. Bordia, H. P. Lüschen, M. H. Fischer, R. Vosk, E. Altman, U. Schneider, and I. Bloch, Observation of Many-Body Localization of Interacting Fermions in a Quasirandom Optical Lattice, Science 349, 842 (2015).

[95] J. Smith, A. Lee, P. Richerme, B. Neyenhuis, P. W. Hess, P. Hauke, M. Heyl, D. A. Huse, and C. Monroe, ManyBody Localization in a Quantum Simulator with Programmable Random Disorder, Nat. Phys. 12, 907 (2016).

[96] J.-y. Choi, S. Hild, J. Zeiher, P. Schauß, A. Rubio-Abadal, T. Yefsah, V. Khemani, D. A. Huse, I. Bloch, and C. Gross, Exploring the Many-Body Localization Transition in Two Dimensions, Science 352, 1547 (2016).

[97] K. Sacha, Modeling Spontaneous Breaking of TimeTranslation Symmetry, Phys. Rev. A 91, 033617 (2015).

[98] T. Shirai, J. Thingna, T. Mori, S. Denisov, P. Hänggi, and S. Miyashita, Effective Floquet-Gibbs States for Dissipative Quantum Systems, New J. Phys. 18, 053008 (2016).

[99] E. H. Lieb and D. W. Robinson, The Finite Group Velocity of Quantum Spin Systems, Commun. Math. Phys. 28, 251 (1972).

[100] B. Nachtergaele and R. Sims, Lieb-Robinson Bounds and the Exponential Clustering Theorem, Commun. Math. Phys. 265, 119 (2006). 